\title{
Network organization during probabilistic learning via taste outcomes
}

Jennifer R Sadler ${ }^{1}$, Grace E Shearrer ${ }^{1,2}$, Nichollette T Acosta ${ }^{1}$, Afroditi Papantoni ${ }^{1}$, Jessica R

Cohen $^{2,3}$, Dana M Small ${ }^{4}$, Soyoung Q Park ${ }^{5,6,7}$, Penny Gordon-Larsen ${ }^{1,8}$, Kyle S Burger ${ }^{1,2}$

1. Department of Nutrition, Gillings School of Global Public Health, University of North Carolina at Chapel Hill, Chapel Hill, NC

2. Biomedical Research Imaging Center, University of North Carolina at Chapel Hill, Chapel Hill, NC

3. Department of Psychology, College of Arts and Sciences, University of North Carolina at Chapel Hill, Chapel Hill, NC

4. Department of Psychiatry, Yale University School of Medicine, New Haven, CT

5. Department of Decision Neuroscience and Nutrition, German Institute of Human Nutrition (DIfE), Potsdam-Rehbruecke, Germany

6. Charité-Universitätsmedizin Berlin, Corporate member of Freie Universität Berlin, Humboldt-Universität zu Berlin, and Berlin Institute of Health, Neuroscience Research Center, Berlin, Germany

7. Deutsches Zentrum für Diabetes, 85764 , Neuherberg, Germany

8. Carolina Population Center, University of North Carolina at Chapel Hill, Chapel Hill, NC

\section{Corresponding Author:}

Jennifer R Sadler

jengilb@ad.unc.edu

2223 McGavran Greenberg Hall

135 Dauer Drive

Chapel Hill NC 27516

Abstract Word Count: 255

Manuscript Word Count: 6550

Funding: This work was supported by NIH R01DDK112317 and the American Psychological Foundation. 


\section{ABSTRACT}

Reinforcement learning guides food decisions, yet how the brain learns from taste in humans is not fully understood. Existing research examines reinforcement learning from taste using passive condition paradigms, but response-dependent instrumental conditioning better reflects natural eating behavior. Here, we examined brain response during a taste-motivated reinforcement learning task and how measures of task-based network structure were related to behavioral outcomes. During a functional MRI scan, 85 participants completed a probabilistic selection task with feedback via sweet taste or bitter taste. Whole brain response and functional network topology measures, including identification of communities and community segregation, were examined during choice, sweet taste, and bitter taste conditions. Relative to the bitter taste, sweet taste was associated with increased whole brain response in the hippocampus, oral somatosensory cortex, and orbitofrontal cortex. Sweet taste was also related to differential community assignment of the ventromedial prefrontal cortex and ventrolateral prefrontal cortex compared to bitter taste. During choice, increasing segregation of a community containing the amygdala, hippocampus, and right fusiform gyrus was associated with increased sensitivity to punishment on the task's posttest. Further, healthy BMI was associated with differential community structure compared to overweight and obese BMI, where high BMI reflected increased connectivity of visual regions. Together, results demonstrate that network topology of learning and memory regions during choice is related to avoiding a bitter taste, and that BMI is associated with increased connectivity of area involved in processing external stimuli. Network organization and topology provides unique insight into individual differences in brain response to instrumental conditioning via taste reinforcers.

Key Words: reward, punishment, taste, modularity, graph theory, brain, fMRI 
GRAPHICAL ABSTRACT

Choice

$\begin{gathered}\text { Community } \\ \text { Segregation }\end{gathered}=\hat{\begin{array}{c}\text { Able to Avoid } \\ \text { Punishment }\end{array}}$ $\underline{\text { Sweet Taste }}$

Bitter Taste
.

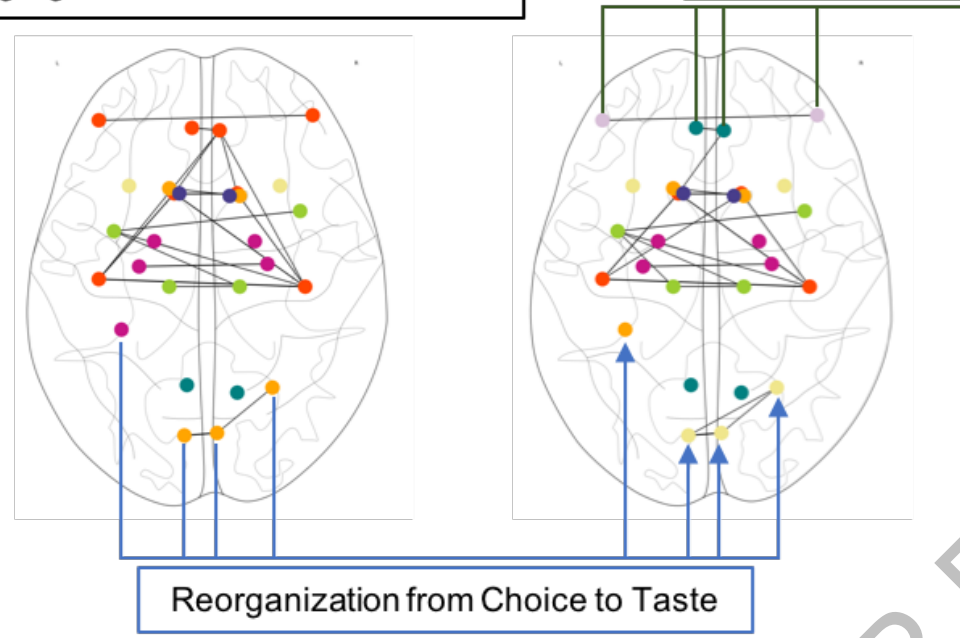




\section{INTRODUCTION}

Overweight and obesity affects over two thirds of American adults [1], making it a major preventable risk factor for a number of diseases including type 2 diabetes [2-4], certain types of cancer [5,6], and cardiovascular disease [7-9]. A variety of individual, social, and environmental factors contribute to the development of overweight and obesity, but eating behavior is considered a key point of study for understanding the etiology of obesity [10]. Eating behavior is the product of repeated choice outcome pairings and the food selected provides the reinforcement. When making a choice about what to eat, information from internal states [11], food preferences [12], dietary goals [13], and the environment [14,15] are integrated in the brain, which selects the best action to perform a motivated action (e.g. satiating hunger, satisfying a craving, or following a diet). These decisions are represented by increased brain response in regions across the prefrontal and cingulate cortices [16]. Regions include the medial orbitofrontal cortex (mOFC), which encodes the value of an outcome [17], and the anterior cingulate cortex (ACC), a region that assesses the cost of actions [18]. Decision making also involves the ventromedial prefrontal cortex (vmPFC), which weighs the value of an outcome against the costs associated with the action to assign overall value to actions $[19,20]$ and the dorsolateral prefrontal cortex (dIPFC), a region that modulates computations [20].

Once a choice is made, consuming the food selected provides initial information about the reinforcing properties of foods via the generation of flavor perceptions, varying from delicious to disgusting. Sweetness, an indicator of the potential presence of energy [21], is generally found to increase pleasantness, whereas bitterness indicates the potential presence of a toxin [22] and, at least initially, promotes dislike. Both sweet and bitter taste therefore serve as important sensory signals to promote or prevent future consummatory behavior [23]. Postoral signals are generated following consumption and update acceptability based on beneficial or adverse ingestive consequences [16]. This process of repeated food choice, consumption, and reinforcement can be operationalized as instrumental conditioning [24]. Following the instrumental conditioning framework: individuals learn to associate a cue (food image or logo) with a given response (e.g. choosing or avoiding the food), and the subsequent reinforcement from consuming the food. This process recruits aforementioned decision-making brain regions, and integrates signal from areas involved in motivation and taste processing [16]. Neural response to taste is well characterized - palatable taste evokes response in the striatum, insula, dorsal ACC, OFC, and amygdala [25,26], while bitter taste also activate the OFC and amygdala [27,28]. 
Typical analyses of functional neuroimaging data provide information about how blood oxygen-level dependent (BOLD) response in the brain changes in response to a given stimulus, but does not capture how areas the brain interact in response to the stimulus. Cognition and behavior are the product of a coordinated flow of information between brain areas, and networkbased approached provide important detail on the functional organization of the brain during response to a stimulus [29]. Functional connectivity is undirected, and can exists between spatially distinct regions, theoretically reflecting synchronous response. For example, functional connectivity is increased between the ventral striatum, insula, amygdala and hippocampus during reinforcement via monetary gain [30]. In response to a palatable taste reinforcer, functional connectivity of the OFC and insula is increased [31]. Network-based analyses of BOLD response apply graph-theory to functional connectivity and provide important information about the architecture of brain networks, and can be applied to identify differences in the functional organization of brain response across conditions or individuals [32,33]. Regions of the brain are treated as "nodes" of a graph and the correlations between nodes (brain regions) as "edges" [34]. Metrics include the organization of brain regions into functional subnetworks that are theorized to perform specialized task (modularity); the density of connections of a region and its neighbors (segregation); the centrality of a region in paths between other area (hubness), and the ratio of with-community connections to out-community connections (integration) (Figure 1) [35]. During reinforcement and motor learning, the communities reorganize, such that the communities become more segregated with learning, supporting the development of specialized function within segregated subnetworks [36,37] Network organization the basal ganglia is particularly important in this process [38]. To date, brain network organization during food reinforcement has not been tested.

Figure 1: Graph Theory Measures of Functional Network Organization

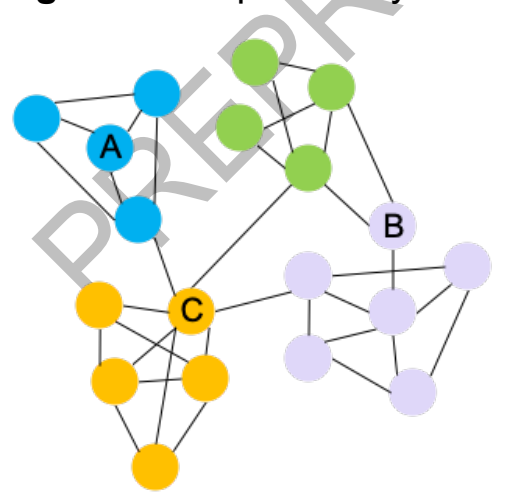

\begin{tabular}{cccc}
\hline Characteristic & Measure & Description & $\begin{array}{c}\text { Node with } \\
\text { High Value }\end{array}$ \\
\hline Segregation & $\begin{array}{c}\text { Clustering } \\
\text { Coefficient }\end{array}$ & $\begin{array}{c}\text { The number of connected } \\
\text { triangles compared to } \\
\text { possible triangles }\end{array}$ & A \\
Integration & $\begin{array}{c}\text { Participation } \\
\text { Coefficient }\end{array}$ & $\begin{array}{c}\text { The ratio of in-community } \\
\text { connections to out- } \\
\text { community connections }\end{array}$ & B \\
Hubness & $\begin{array}{c}\text { Betweenness } \\
\text { Centrality }\end{array}$ & $\begin{array}{c}\text { The number of shortest } \\
\text { paths between nodes that } \\
\text { travel through that node }\end{array}$ & C \\
\hline
\end{tabular}

Community 1

Community 2

Community 3

Community 4 
The extant studies on brain response during food reinforcement primarily use passive, Pavlovian conditioning tasks, where no choice is involved. Pavlovian conditioning and instrumental conditioning via secondary reinforcers are associated with differences in BOLD response [39], so it stands to reason that brain response during taste-mediated instrumental conditioning may diverge from prior response to passive conditioning tasks. Additionally, tasks assessing brain response during instrumental conditioning primarily use secondary reinforcers such as money or written feedback. A primary reinforcer, such as food, may have more saliency [40] and could contribute to different network organization and dynamics over learning [41]. Thus, to address gaps in our understanding of brain response and network structure during instrumental conditioning, this study tested brain response during completion of a responsedependent conditioning task with taste reinforcement. We adapted a canonical probabilistic learning task, which measures learning from reward and punishment [42], by using beverages as reinforcement. The primary aim of the study was to identify whole brain response and explore network organization using a priori regions of interest (ROI) based analysis during the task, specifically in the choice, sweet taste, and bitter taste conditions. We hypothesized that choice would be associated with BOLD response in the medial OFC, vmPFC, and dIPFC, that sweet taste would be associated with response in the insula, dorsal ACC, OFC, and amygdala response, and bitter taste would be associated with response in the OFC and amygdala. For network analyses, we hypothesized that choice will be associated with a fewer number of communities than reward and punishment, reflecting more synchronization across the brain during choice. In addition to these aims, we also tested if measures of network segregation, hubness, and integration were connected to behavioral outcomes including task performance and preference ratings of the sweet and bitter beverages. We hypothesized that increased community segregation across conditions would be associated with improved task performance as measured by posttest accuracy.

\section{MATERIALS AND METHODS}

2.1. Sample. Ninety $(n=90)$ male and female participants were recruited from the Chapel Hill, North Carolina area to complete a cross sectional study. Eligibility criteria included: 1) age 18-28 years, 2) body mass index between (BMI) $20.0 \mathrm{~kg} / \mathrm{m}^{2}$ and $32.0 \mathrm{~kg} / \mathrm{m}^{2}$. Exclusion criteria were: 1) contraindications of MRI (e.g. metal implants, piercings, pregnancy), 2) current smoking, 3) selfreported current or past diagnoses of an eating disorder, 4) chronic illness or medication requirement that could affect diet, 5) diagnosis of a major psychological condition (bipolar, schizophrenia, major affective disorder), and 6) allergy or intolerance to any study foods. The 
Institutional Review Board of the University of North Carolina at Chapel Hill approved all methods and study participants gave written consent before the start of testing. For this analysis, three $(n=3)$ participants who ended scanning early and two $(n=2)$ participants who had $<29$ trials (representing $56 \%$ of possible trials) of reward or punishment were excluded. The resulting analytic sample was $n=85$ participants.

2.2. Measures. Participants completed all measures in a 2.5 hour visit at the University of North Carolina at Chapel Hill's Gillings School of Global Public Health and Biomedical Research Imaging Center (BRIC). Participants were instructed to fast for four hours before the visit to standardize time since last meal. Visits took place within the hours of 7:00 and 20:00.

Participants were instructed to fast for at least 4 hours before their assessment.

2.2.1. Anthropometrics \& Demographics. Height (to the nearest $0.5 \mathrm{~cm}$ ) and weight (to the nearest $0.1 \mathrm{~kg}$ ) were measured with a wall-mounted stadiometer and a calibrated scale by trained research staff. BMI was calculated as $\mathrm{kg} / \mathrm{m}^{2}$. Demographics including age, race, and ethnicity were assessed via self-report.

2.2.2. Beverages and Preference Assessment. Beverages used in the instrumental conditioning task as reward and punishment were selected based on participant ratings via a taste test. During the taste test, participants were instructed to taste and rate $20 \mathrm{~mL}$ samples of 4 sweet and 4 bitter beverages. The eight beverages were made from a base of water $(940 \mathrm{~mL})$, unsweetened Kool-Aid® Cherry powder $(4.5 \mathrm{~g})$ and simple syrup (60 mL). Simple syrup or a quinine solution were added to the beverages to create different levels of sweetness or bitterness. The composition of the beverages can be seen in Table 1. The beverages were calorically-matched with the addition of maltodextrin, a soluble, neutral-tasting carbohydrate powder. Levels of sweetness and bitterness were selected from previous studies of taste preference [43]. Beverages were rated in pleasantness, desire to consume, sweetness, bitterness and intensity on VAS anchored at -100 and 100 (sample anchors were stated as 'most imaginable' and 'least imaginable' with 0 identified as neutral). All sweet beverages were sampled in a random order, then participants ranked the beverages from most pleasant to least pleasant, with the highest ranked beverage selected as the reward for the probabilistic selection task (see 2.2.4). The same process was then completed with the bitter beverages, and the lowest ranked beverage was selected as the punishment. 
Table 1. Reinforcer Composition and Selection

\begin{tabular}{lccccc}
\hline Flavor & kcal $^{1}$ & $\begin{array}{c}\text { Sugar } \\
(\mathbf{g})^{\mathbf{1}}\end{array}$ & $\begin{array}{c}\text { Quinine } \\
(\mathbf{m g})^{1}\end{array}$ & $\begin{array}{c}\text { Malto- } \\
\text { dextrin }(\mathbf{g})^{1}\end{array}$ & $\begin{array}{c}\text { Selected as } \\
\text { Reinforcer }\end{array}$ \\
\hline Sweet 1 & 104.6 & 14.2 & -- & 20.9 & $11(12.9 \%)$ \\
Sweet 2 & 105.4 & 21.2 & -- & 10.5 & $17(20.0 \%)$ \\
Sweet 3 & 105.4 & 28.2 & -- & 5.3 & $20(23.5 \%)$ \\
Sweet 4 & 105.4 & 35.1 & -- & 0.0 & $37(43.5 \%)$ \\
\hline Bitter 1 & 105.3 & 7.0 & 12.0 & 7.0 & $5(5.9 \%)$ \\
Bitter 2 & 105.3 & 6.8 & 24.0 & 7.1 & $11(12.9 \%)$ \\
Bitter 3 & 105.3 & 6.3 & 48.0 & 7.2 & $19(22.4 \%$ \\
Bitter 4 & 105.3 & 5.5 & 72.0 & 7.4 & $50(58.8 \%)$ \\
\hline 1Values reported for 300mL portion of each beverage \\
\hline
\end{tabular}

2.2.3. Neuroimaging Methods. Anatomical and functional imaging data were collected in a Siemens Prisma 3T scanner (Siemens Medical Solutions, Munich, Germany) at UNC's Biomedical Research Imaging Center (BRIC). Visual stimuli were presented with a digital projector/reverse screen display system. Tastants were delivered using programmable syringe pumps (Braintree Scientific BS-8000, Brain-Tree, MA) operated through a program written in PsychoPy [44], available at: https://github.com/niblunc/bevel task) to ensure consistent volume, rate, and timing of taste delivery. A set of tubing attached to the scanner bed was placed into the participants' mouths and delivered the tastes. Button press response was collected via a 5button response pad (Current Designs Pyka Response Pad, Philadelphia, PA) held in the participant's right hand. Blood-oxygen-level-dependent (BOLD) signal was collected during functional runs under the following scanning parameters: $T R=2000 \mathrm{~ms}$, $T E=20 \mathrm{~ms}$, flip angle $=$ $80^{\circ}$, with a spatial resolution of $3.0 \mathrm{~mm}$. Images were collected with whole-brain coverage; 32 $4 \mathrm{~mm}$ slices (interleaved acquisition) were acquired along the AC-PC transverse, oblique plane as determined by the midsagittal section. Anatomical scans were acquired with a TR/TE of $2100 \mathrm{~ms} / 2.4 \mathrm{~ms}$, flip angle of $15^{\circ}$, TI of $1100 \mathrm{~ms}$, matrix size of $256 \times 256$, FOV of $22 \mathrm{~cm}$, and slice thickness of $1 \mathrm{~mm}$.

2.2.4. Appetitive Probabilistic Selection Task. Participants completed a modified, taste-based version of the Probabilistic Selection Task [42], measuring response to reward and punishment (Figure 2). The task was composed of training and posttest phases. In the training phase, participants were presented with pairs of novel shapes, and asked to select the "correct" shape 
to receive a sweet taste. Participants were instructed that when they chose "incorrectly", they would receive a bitter taste. Feedback was probabilistic; each shape was reinforced at a prespecified probability. Three pairs were presented during the scan: 1) AB pair (A: $80 \%$ correct; B: $20 \%$ correct); 2) CD pair (C: 70\% correct, D: 30\% correct); 3) EF pair (E: 60\% correct, F: $40 \%$ correct). Instances where participants selected the higher probability shape but received a bitter taste or where participants selected the lower probability shape but received a sweet taste were classified as "prediction errors". Pairs were presented in a random, intermixed order. Taste feedback ( $3 \mathrm{~mL}$ of sweet taste or $3 \mathrm{~mL}$ of bitter taste) was delivered over 5 seconds in the absence of any visual stimuli then followed by a $1 \mathrm{~mL}$ rinse of a tasteless solution made to mimic the taste of saliva, delivered over 2 seconds. The next trial proceeded following a 3-7 second variable gap, during which a fixation cross was shown. In total, participants completed 104 training trials over four runs, each 6 minutes and 44 seconds in length. Following the training phase during the fMRI scan, participants completed the posttest phase of the task outside of the scanner. During the posttest, participants were presented with one shape from the $A B$ set $(A: 80 \%$ correct, $B: 20 \%$ correct) paired with shapes from the other two sets and asked to select the shape that is more likely to be "correct". The proportion of trials in which the participant selects the A shape is considered their sensitivity to reward, and the proportion of trials in which the participant avoids the $B$ shape is considered their sensitivity to punishment.

2.3 Appetitive Probabilistic Selection Task Performance. Statistical analyses of task data were carried out using the R statistical software package (Version 3.5.1, R Foundation for Statistical Computing, Vienna, Austria). PST task performance was calculated as the percent of correct choices, defined as choosing the shape with the higher likelihood of being correct in each pair. The percent of $A B$ trials where $A$ was selected, the percent of $C D$ trials where $C$ was selected, and the percent of EF trials where $E$ was selected were calculated over the 4 training blocks, representing participant's training accuracy (Figure 2B). Repeated measures ANOVA was implemented using the 'Ime4' package ( $v$ 1.1) to test for significant change in task performance by pair over runs, accounting for intra-individual correlation. Posttest accuracy was calculated as the percent of correct choices, defined by selecting the A shape (sensitivity to reward), and avoiding the $B$ shape (sensitivity to punishment). Posttest performance was correlated with brain response as a continuous regressor and categorically (See 2.7). For categorical analyses, participants were categorized as 'learners' if their posttest accuracy was above chance (50\%), and as 'non-learners' if their posttest accuracy was below chance. 
2.4 FMRI Preprocessing. Neuroimaging data were preprocessed using the fMRIPrep pipeline [45]. In brief, DICOMS were converted to the Brain Imaging Data Structure (BIDS file structure) [46], then preprocessed using fMRIPrep. FMRIPrep preprocessing included skull stripping using Advanced Normalization Tools (ANTs); tissue segmentation using FSL's Automated Segmentation Tool (FAST); and spatial normalization to Montreal Neurological Institute (MNI) 152-Asymmetrical space using ANTs' registration option. FreeSurfer was used to reconstruct surfaces from structural images. Functional images were then motion corrected using FSL's MCFLIRT, corrected for fieldmap distortion, and spatially smoothed using a $6 \mathrm{~mm}$ Gaussian full width half maximum isotropic kernel. In FSL, final preprocessing included adjusting for autocorrelation and highpass filtering and adjusting for nuisance regressors, including the 6 motion parameters, their derivatives, and high motion time points $(>0.9)$.

2.4 FMRI Group-Level Main Effects Analysis. Neuroimaging analyses were primarily completed in FSL (FMRIB Software Library, www.fmrib.ox.ac.uk/fsl) Individual and group level analyses were carried out in FSL's FMRI Expert Analysis Tool (FEAT; [47,48]. At the individual level, within subject models assessed brain response to 1) choice via button press > baseline; 2) reward via sweet taste > rinse; and 3) punishment via bitter taste > rinse. Individual level contrasts also included reward > punishment; and punishment > reward. Motion parameters were included as nuisance regressors at the individual level.

At the group level, the analysis followed a one sample F-test model to identify whole brain response that was significantly different within the full sample. Main effect models were examined for the following contrasts: 1) choice > baseline (fixation cross); 2) sweet taste > tasteless solution; 3 ) bitter taste > tasteless solution; 4) sweet taste > bitter taste; and 5) bitter taste > sweet taste. Multiple comparisons were controlled for by using the threshold free cluster enhancement, nonparametric thresholding algorithm in FSL's Randomise (n permutations = 5000) resulting in a family-wise error rate corrected significance threshold of $p_{F W E}<0.05$ [49]. Localization of significant clusters were determined using Mango's MNI atlas tool.

2.5 Betaseries and Functional Connectivity. A betaseries regression analysis was used to measure condition-specific functional connectivity in the sample [50]. In each participant's preprocessed data, separate general linear models were set with a regressors modeling each event and another regressor modeling all other events of the same condition to derive conditionwise whole brain beta values for each event [51]. Conditions included: 1) choice and button press; 2) reward; 3) punishment. The resulting beta images were concatenated by condition and 
run, producing a timeseries of beta values, or betaseries for each condition and for each participant. Then, average signal was extracted from each participant's betaseries in 28 regions of interest (ROIs) drawn from the Big Brain 300 parcellation, a functional parcellation based on the Power atlas [52], with improved coverage of subcortical regions [53]. ROls were selected based on regions reported as responsive to taste stimuli, taste administration, and reinforcement learning [54-58] (Table 4). The resulting betaseries arrays represented average parameter estimates in each $\mathrm{ROI}$ for each event in a given condition. Matrices were the same size $(28 \times 104)$ in the choice condition. Because reinforcement varied, reward and punishment matrices varied in length from $28 \times 29$ to $28 \times 64$. Betaseries correlation matrices for the 28 ROls were extracted, resulting in $28 \times 28$ betaseries connectivity matrix for each condition, for each participant. For methods and results of significance testing of functional connectivity (ROI - ROI correlation), see Supplemental Materials.

2.6 Graph construction \& network topology. To examine network topology during choice, reward, and punishment, betaseries correlation were used as the basis of network graphs. The 28 ROls were treated as nodes, and correlations between ROls were treated as graph edges. For graph analysis, only positive correlations were included, since network topology measures are not optimized for negative correlations [59]. To generate graphs, participants' correlation matrices were fed into 'networkX' package (version 2.3) [60] and 'bctpy' package (version 0.5.1, https://pypi.org/project/bctpy/), implemented in Jupyter Notebook [61], running Python 3.7.5 (Python Software Foundation).

Within each participant's graph, modularity, or the degree to which the network may be subdivided into such clearly delineated groups called communities, was calculated using the Louvain algorithm [62] 'python-louvain' package (version 0.13). Modularity was calculated on the median graph, rather than the mean graph, to account for skewness. Then the median graph was partitioned into the identified communities, and the weighted edges between communities were calculated to examine the connectivity between communities.

Measures of network topology were also computed for each node within participant's graphs, then metrics were averaged across all nodes in the communities identified at the group level, producing average community parameters for networks associated with choice, reward, and punishment. Three parameters were calculated: (1) Weighted clustering coefficient, or the geometric average of the subgraph edge weights, was used to assess segregation via networkX's 'cluster' algorithm [63]. Higher clustering coefficient values suggest greater separation of a node from nodes outside its module. (2) Weighted betweenness centrality which 
measures community hubness by the fraction of all shortest paths in the network that contain a given node, and calculated using via networkX's 'betweenness_centrality' algorithm [64]. Higher betweenness centrality suggests increased participation in paths between other nodes, and higher hubness. (3) Participation coefficient, or the ratio of within-community connections compared to between-community connections, measures how integrated a node is within its community and the network. Participation coefficient was calculated using bctpy's 'participation_coef' algorithm [65]. Participation coefficients closer to 1 are indicative of greater within-community connectivity, while participation coefficients closer to 0 represent greater between community connectivity. For each participant's graph, clustering coefficient, betweenness centrality, and participation coefficient was calculated at the node level, then averaged across communities. All analyses were performed in non-thresholded, weighted graphs. To visualize modularity and nodal connectivity results, a mean graph of all conditions was created, then thresholded at the average edge strength $(>0.30)$ to display strongest edges.

\subsection{Associations between network topology and participant characteristics.}

To examine differences in network structure associated with participant characteristics, we tested for group-based differences in network organization on three categorical variables: BMI, sex, and posttest performance. To examine differences in network structure between BMI groups, sample was separated into healthy BMI $\left(n=52 ; B M I<25.0 \mathrm{~kg} / \mathrm{m}^{2}\right)$ and overweight/obese $\mathrm{BMI}\left(\mathrm{n}=33 ; \mathrm{BMI}>25.0 \mathrm{~kg} / \mathrm{m}^{2}\right)$. Modularity was then calculated in the mean healthy BMI graph and mean overweight/obese BMI graph. Community organization was compared between male $(n=42)$ and female $(n=43)$ participants, and participants who scored above $50 \%$ on the appetitive PST posttest $(n=45)$ and those who scored below $50 \%(n=40)$. For each pair of groups, differences in community assignment were compared qualitatively and quantitatively using the normalized mutual information (NMI) score ([66], similar methods as [67]). NMI scores range from 0 to 1 with higher scores reflecting more similar community assignment between groups. To identify empirically meaningful differences in network organization, we compared observed NMI scores to a distribution of null NMI scores generated by randomly splitting individual matrices into null groups and comparing community organization. Null NMI scores were calculated over 500 permutations to generate a null distribution of NMI values for each contrast. Observed NMI values that exceeded one standard deviation of the mean of the null distribution, were considered a quantitative measure of meaningful differences in community organization. 
Further, we tested for associations between community metrics (segregation, integration, and hubness) and behavioral outcomes including stimuli ratings, the level of sweetness/bitterness and posttest performance in R statistical software package (version 3.5.1, R Foundation for Statistical Computing, Vienna, Austria). Tests included Pearson's productmoment correlation for continuous measures (pleasantness and desire VAS ratings, posttest sensitivity to reward, posttest sensitivity to punishment) and one-way analysis of variance (ANOVA) for categorical measures (sweetness of reward selected and bitterness of punishment selected) using the 'Imer' function from the 'Ime4' package [68]. Significance was considered at multiple-comparisons corrected $p$-value threshold $<0.008$.

\section{RESULTS}

3.1 Participant Characteristics. Participants characteristics and performance on the instrumental conditioning task are summarized in Table 2. The sample was primarily young, healthy-weight adults. The majority of participants identified as white and non-Hispanic. Just over half of the participants were female.

Table 2: Participant Characteristics and Task Performance $(n=85)$

\begin{tabular}{lcc}
\hline & Mean \pm SD & Min - Max \\
Age (years) & $21.5 \pm 2.4$ & $18-28$ \\
BMI $\left(\mathrm{kg} / \mathrm{m}^{2}\right)$ & $24.7 \pm 3.2$ & $19.6-33.1$ \\
Pre-scan Hunger & $16.1 \pm 46.1$ & $-100-80$ \\
Sweet Taste Pleasantness & $38.7 \pm 19.1$ & $-32-100$ \\
Bitter Taste Pleasantness & $-45.5 \pm 25.8$ & $-96-19$ \\
Sex & Count & Percent \\
$\quad$ Male & 42 & $49.4 \%$ \\
$\quad$ Female & 43 & $50.6 \%$ \\
Race & & \\
$\quad$ Black or African American & 4 & $4.7 \%$ \\
$\quad$ Asian or Pacific Islander & 19 & $22.4 \%$ \\
$\quad$ White & 51 & $60.0 \%$ \\
$\quad$ Middle Eastern & 2 & $2.4 \%$ \\
$\quad$ More than one race & 5 & $5.9 \%$ \\
$\quad$ Other or chose not to report & 4 & $4.7 \%$ \\
Ethnicity & \multicolumn{3}{c}{} \\
$\quad$ Hispanic & 10 & $11.8 \%$ \\
$\quad$ Non-Hispanic & 75 & $88.2 \%$ \\
\hline PST Performance & Mean \pm SD & Min - Max \\
Training Rewards (n) & $49.8 \pm 6.0$ & $29-63$ \\
Training Punishments (n) & $48.4 \pm 5.7$ & $31-64$ \\
Prediction Error Trials (\%) & $27.8 \pm 4.4$ & $18.4-38.2$ \\
Posttest Sensitivity to Reward & $51 \% \pm 10.6 \% 29.2 \%-75.0 \%$ \\
& &
\end{tabular}


Posttest Sensitivity to Punishment $50.8 \% \pm 9.3 \% 29.4 \%-83.3 \%$

3.2 Appetitive Probabilistic Selection Task Performance. Out of the 104 training trials in the modified Probabilistic Selection Task, participants responded to receive reinforcement on an average of $98.1 \pm 5.6$ trials. About half of those trials were reinforced with the reward (sweet taste, mean $=49.8 \pm 6.0$ trials), while participants also received a high number of punishment reinforcers (bitter taste, mean $=48.4 \pm 5.7$ trials). Average accuracy did not increase over course of training (Figure 2B). On the PST posttest, participants chose shape A on $51.0 \pm$ $11.0 \%$ of trials, representing their sensitivity to food reward (Table 2; Figure 2C). Participants avoided shape $B$ on $51.0 \pm 9.0 \%$ of trials, representing their sensitivity to food punishment. The correlation between sensitivity to reward and punishment was low $(r=0.135)$. Based on posttest performance, 45 participants were able to choose shape $A$ and avoid shape $B$ on over $50 \%$ of trials, classifying as 'learners', while 40 participants we classified as 'non-learners' as their posttest accuracy was below $50 \%$. There were no significant correlations between posttest performance and individual factors such as BMI, age, VAS ratings of the sweet and bitter tastes, training performance or the percent of prediction error trials (correlation coefficients $(r)$ : $0.002-$ 0.201; see Supplemental Materials for full results), and there were no significant differences between the 'learner' and 'non-learner' groups in the individual factors examined ( $p$ 's: 0.09 0.81 ; see Supplemental Materials for full results). 
Figure 2: Appetitive PST Task and Performance
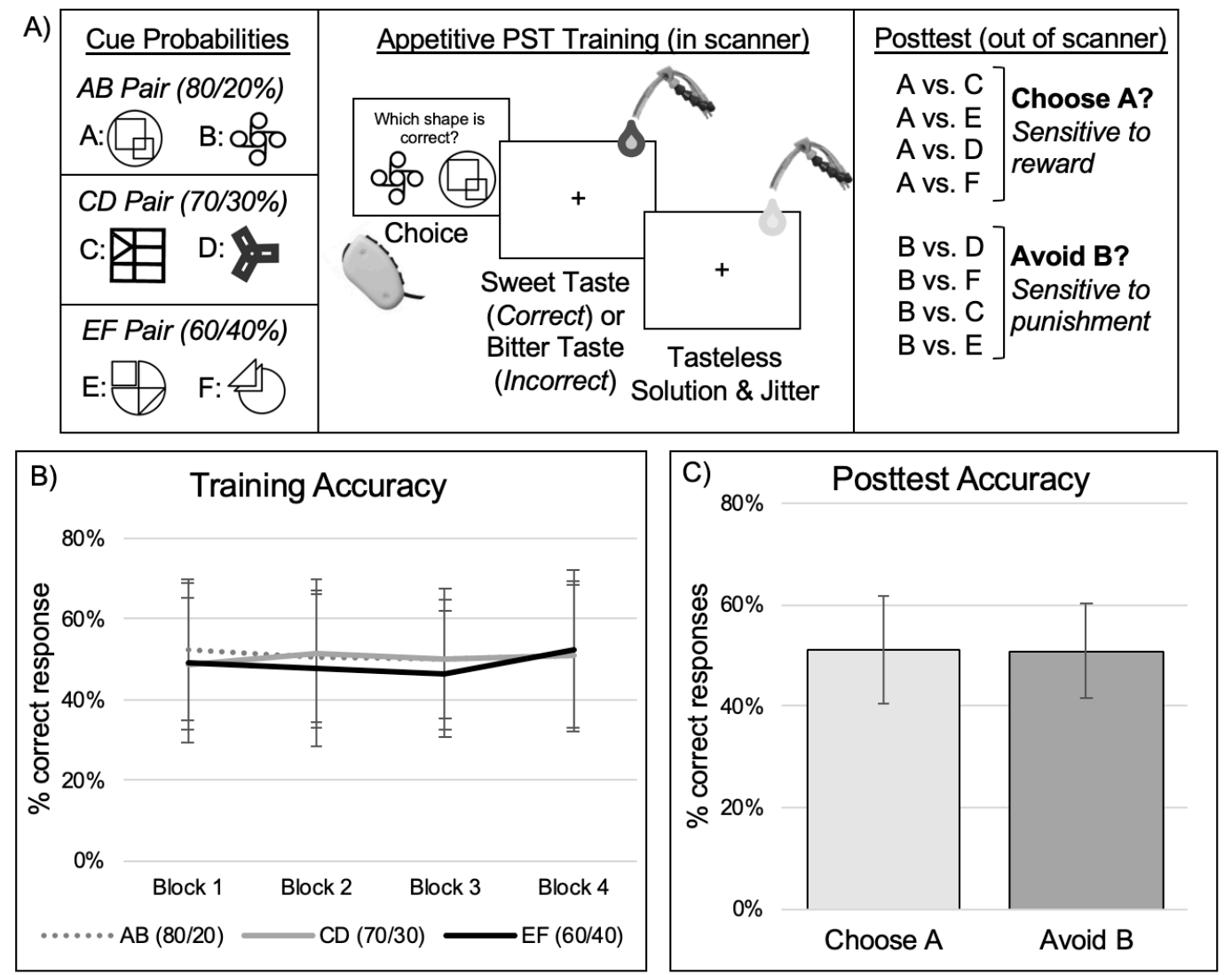

3.3 Brain response during choice, reward, and punishment. We observed robust main effects during choice via button press; sweet taste (reward); and bitter taste (punishment). During choice via button press, BOLD response was observed across the occipital cortex and temporal lobe, insula, primary motor cortex, as well as anterior cingulate cortex, and orbitofrontal cortex (Table 3; Figure 3A). In response to sweet taste (contrasted against rinse), response was found across a number of regions including peaks in the temporal lobe and hippocampus, lateral occipital cortex, and frontal pole, in addition to response in the insula, precuneus, thalamus, left dorsolateral prefrontal cortex, amygdala, and oral somatosensory cortex (Table 3; Figure 3B). In response to bitter taste (contrasted against rinse), a similar pattern of response to reward was found, with peaks in the thalamus, oral somatosensory cortex, and anterior cingulate (Table 3; Figure 3C). When comparing whole-brain response to sweet taste contrasted against bitter taste (sweet taste > bitter taste), robust response extended into regions that traditionally respond to rewarding food stimuli, including response in the oral somatosensory cortex, precuneus, and orbitofrontal cortex (Figure 3D). Sweet taste was also associated with greater response in the hippocampus, dorsal anterior cingulate cortex, and 
caudate. Conversely, bitter taste contrasted against sweet taste (bitter taste $>$ sweet taste) was related with increased response in the paracingulate gyrus (Figure 3D).

Table 3. Significant BOLD Response to Cues and Reinforcement

\begin{tabular}{|c|c|c|c|c|c|c|}
\hline Contrast \& Regions & $k^{1}$ & $\mathbf{Z}$ & pFWE $^{1}$ & $\mathbf{X}^{2}$ & $\mathbf{Y}$ & $\mathbf{Z}$ \\
\hline \multicolumn{7}{|l|}{ Choice $>$ Rest } \\
\hline $\begin{array}{l}\text { Occipital cortex and temporal lobe } \\
\text { (bilateral) }\end{array}$ & 11594 & 11.3 & $<0.001$ & 33 & -48 & \\
\hline Precentral gyrus (bilateral) & 1268 & 9.3 & $<0.001$ & 0 & 15 & \\
\hline Insula $(\mathrm{R})$ & 839 & 9.3 & $<0.001$ & 33 & 27 & 3 \\
\hline Caudate (L) & 144 & 6.8 & $<0.001$ & -9 & 6 & 3 \\
\hline Caudate (R) & 121 & 6.4 & $<0.001$ & 9 & 9 & 6 \\
\hline Supramarginal Gyrus (R) & 94 & 5.1 & $<0.001$ & 60 & -42 & 18 \\
\hline Middle Temporal Gyrus (L) & 57 & 5.1 & $<0.001$ & -54 & -54 & 12 \\
\hline Middle Frontal Gyrus (R) & 30 & 4.4 & 0.006 & 45 & 27 & 24 \\
\hline \multicolumn{7}{|l|}{ Reward (Sweet Taste) > Rinse } \\
\hline Temporal lobe and hippocampus (L) & 10032 & & $<0.001$ & -30 & -36 & -9 \\
\hline Lateral occipital cortex (L) & 152 & & $<0.001$ & -54 & -72 & 33 \\
\hline Frontal Pole (L) & & & 0.011 & -15 & 63 & 33 \\
\hline Frontal Pole (R) & 30 & 4.9 & 0.020 & 6 & 60 & 45 \\
\hline \multicolumn{7}{|l|}{ Punishment (Bitter Taste) > Rinse } \\
\hline Thalamus (L) & & 7.0 & $<0.001$ & -3 & -18 & 0 \\
\hline Oral Somatosensory cortex (L) & 2065 & 6.3 & $<0.001$ & -21 & -33 & 66 \\
\hline Anterior cingulate $(\mathrm{L})$ & 230 & 5.3 & 0.042 & -6 & 39 & -6 \\
\hline $\begin{array}{l}\text { Superior frontal gyrus and paracingul } \\
\text { gyrus (L) }\end{array}$ & 129 & 5.2 & 0.009 & -9 & 12 & 51 \\
\hline \multicolumn{7}{|c|}{$\begin{array}{l}\text { Reward (Sweet Taste) > Punishment (Bitter Taste) } \\
\text { Hippocampus, dorsal ACC, and temporal }\end{array}$} \\
\hline lobe & 10132 & 6.4 & $<0.001$ & -30 & -18 & -15 \\
\hline Oral somatosensory cortex (R) & 99 & 5.0 & $<0.001$ & 54 & -6 & 30 \\
\hline Precentral Gyrus (L) & 66 & 4.4 & 0.013 & -3 & -30 & 78 \\
\hline Orbitofrontal Cortex (R) & 39 & 4.5 & $<0.001$ & 42 & 33 & -18 \\
\hline \multicolumn{7}{|c|}{ Punishment (Bitter Taste) > Reward (Sweet Taste) } \\
\hline Paracingulate Gyrus & 244 & 5.7 & 0.029 & 6 & 12 & 48 \\
\hline
\end{tabular}


Figure 3: Brain Response During Choice, Sweet Taste, and Bitter Taste
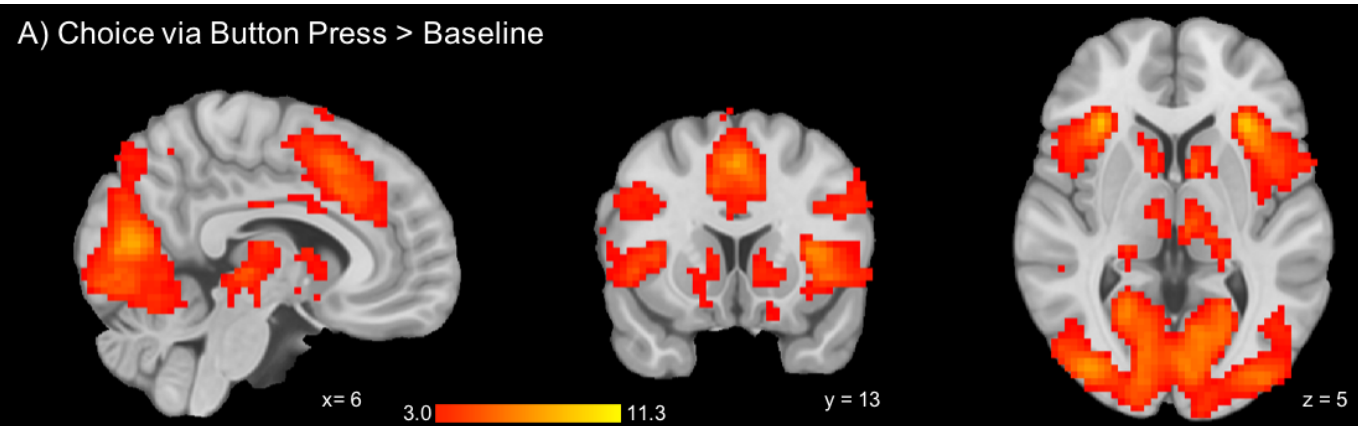

B) Sweet Taste > Tasteless Solution
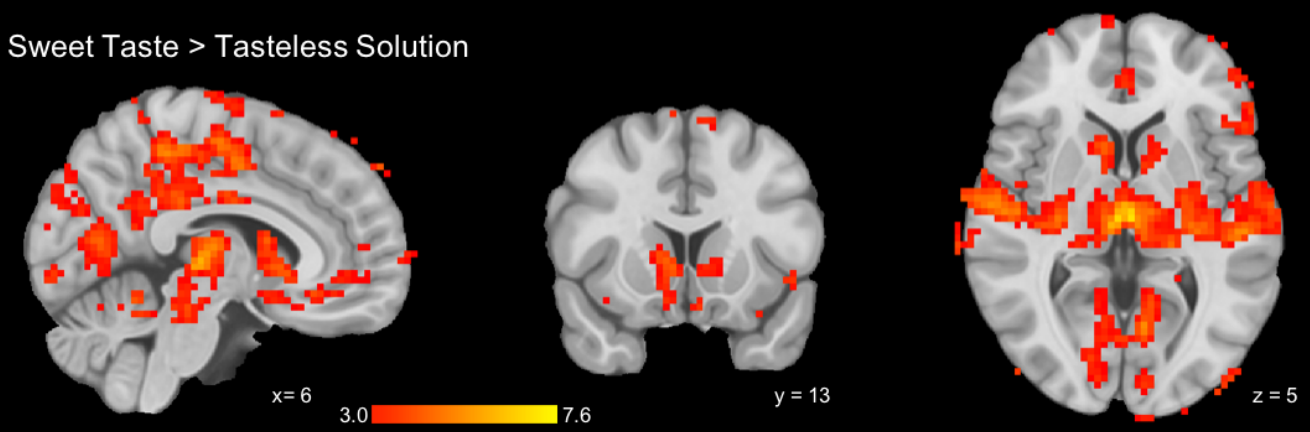

C) Bitter Taste > Tasteless Solution
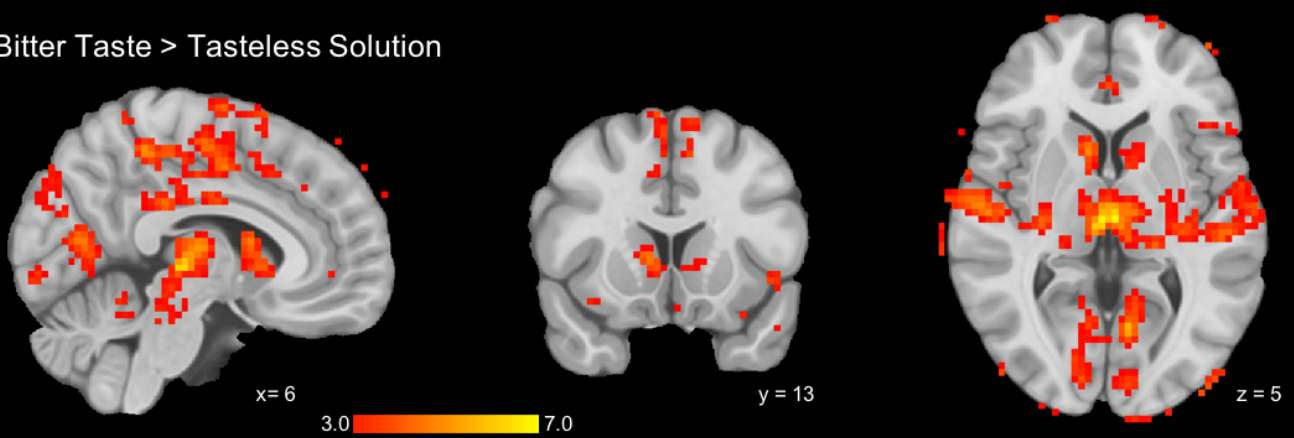

D) Sweet Taste $>$ Bitter Taste (Red) and Bitter Taste $>$ Sweet Taste (Blue)
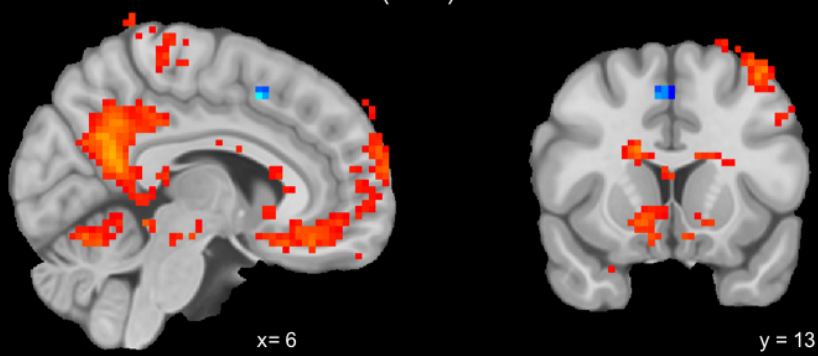

$y=13$

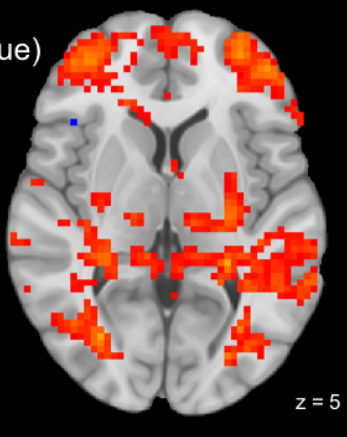

3.0

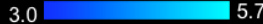

3.4. Network Organization during Choice, Sweet Taste, and Bitter Taste.

Overall community organization is shown in Figure 4. During choice, ROls organized into seven communities (Table 4), including a learning and memory community (1: amygdala, hippocampus, and fusiform gyrus), a motivation, motor response and behavioral control 
community (2: dorsal striatum, pre/postcentral gyrus, ventromedial PFC, and ventrolateral PFC) and a visual response and valuation community (3: medial OFC, intracalcarine cortex, fusiform gyrus). During sweet taste and bitter taste, ROls organized into eight and seven communities respectively (Table 4). Overall, community assignment during bitter taste was similar to sweet taste and choice. However, the ventromedial PFC and ventrolateral PFC were assigned to distinct communities during sweet taste, while during punishment, the ROls were assigned to community 2 (Table 4, Figure 4).

\subsection{Association of Network Topology with Task Performance, BMI and Sex. Differences in} community organization between BMI groups were observed (Figure 5A). During response to sweet taste, ROIs in the bilateral intracalcarine cortex and right fusiform gyrus were organized into a single community in participants with healthy BMI $(n=52)$. Among participants with an overweight or obese BMI $(n=33)$, the ROls were assigned to a larger community including the left fusiform gyrus and bilateral medial OFC. To test if the observed differences were quantitatively meaningful, we calculated a normalized mutual information (NMI) score for the BMI-groups' community assignment, then compared it to a distribution of null scores generated from permutation testing with random group assignment. The observed NMI describing the differences between BMI groups in community organization during sweet taste (0.669) was greater than 1 SD lower than null NMI distribution mean (mean \pm SD: $0.791 \pm 0.102$; Figure 5B), supporting that the observed differences are not due to random variation in assignment.

Segregation of community 1 during choice (amygdala, dorsal striatum, fusiform gyrus) was significantly associated with sensitivity to punishment as assessed on the probabilistic selection task posttest $(r=0.289, \mathrm{t}=2.73, \mathrm{df}=82, p=0.0077$; Figure $5 \mathrm{C})$. Measures of community segregation, integration, or hubness during sweet taste and bitter taste relate to posttest performance (\%sensitivity to punishment or \%sensitivity to reward) or reinforcer characteristics (ratings of pleasantness and desire to consume, sugar content, or quinine added) (p's: $0.016-0.99$ ). Differences in community assignment were observed between posttest accuracy groups as well as between male and female participants, but the differences were not meaningfully different from the null NMI distribution mean, and thus were not considered meaningfully different (Supplemental Materials). 
Figure 4: Network Community Structure during Choice, Reward, and Punishment

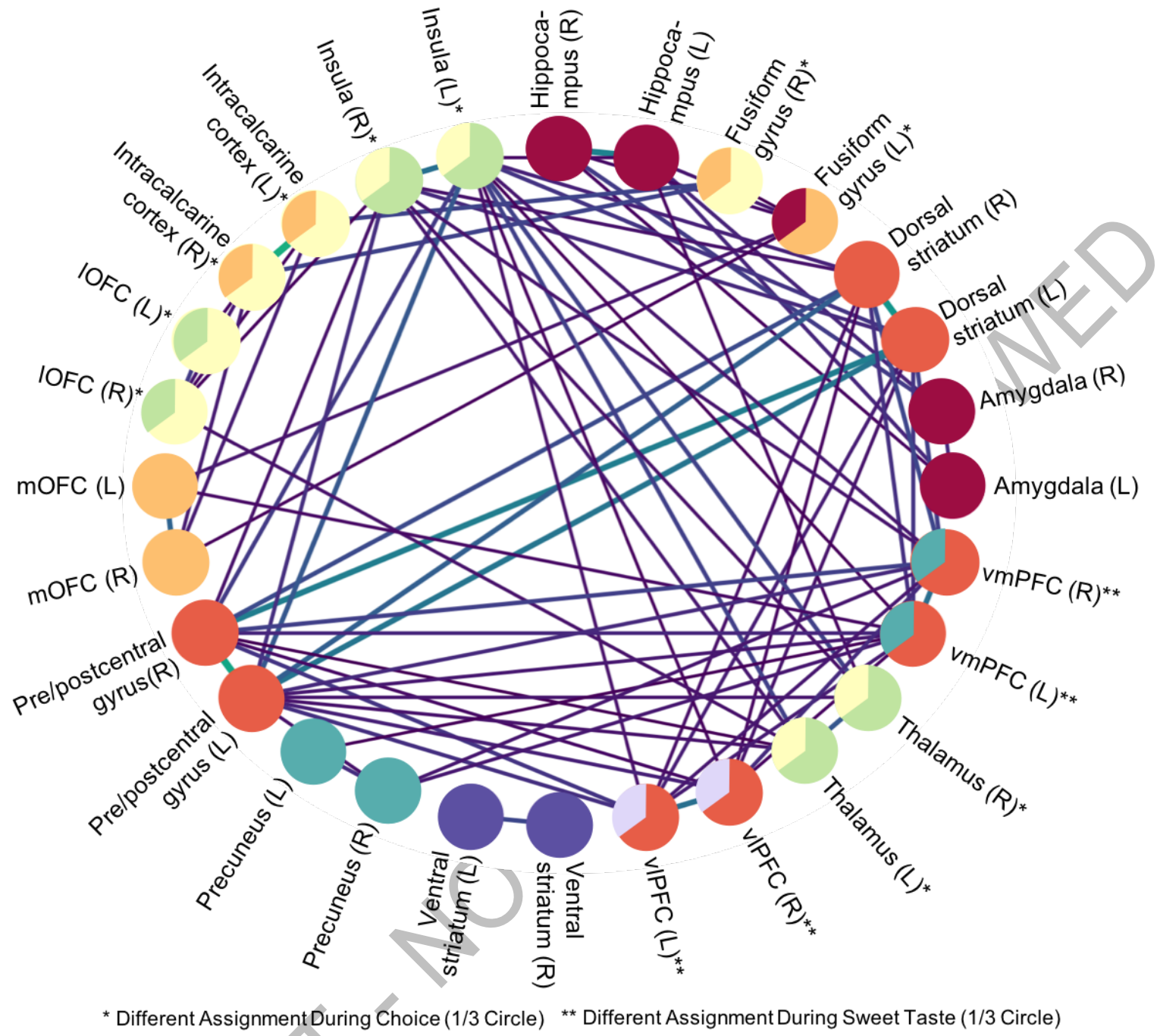

* Different Assignment During Choice (1/3 Circle) ** Different Assignment During Sweet Taste (1/3 Circle)
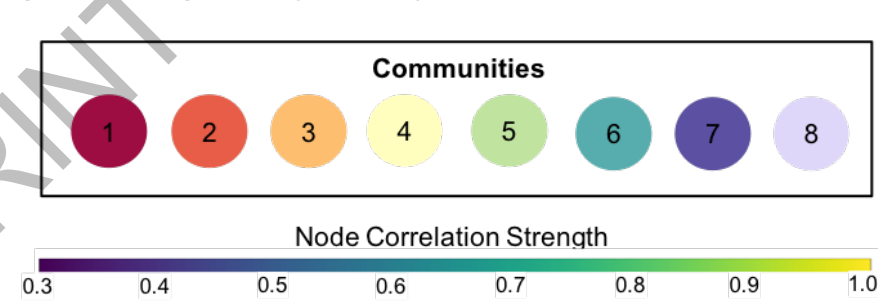
Table 4: Brain Network Communities During Choice, Sweet Taste, and Bitter Taste

\begin{tabular}{|c|c|c|c|c|c|c|c|}
\hline \multirow[b]{2}{*}{ Region } & \multicolumn{3}{|c|}{$\frac{\text { Coordinates }}{(\mathrm{mm})}$} & \multicolumn{3}{|c|}{ Community Assignment } & \multirow{2}{*}{$\begin{array}{l}\text { Unique } \\
\text { Community } \\
\text { Assignment }\end{array}$} \\
\hline & $x$ & $\mathrm{y}$ & z & Choice & Sweet & Bitter & \\
\hline Amygdala (L) & -20 & -2 & -22 & 1 & 1 & 1 & - \\
\hline Amygdala (R) & 20 & -2 & -23 & 1 & 1 & 1 & - \\
\hline Dorsal striatum (L) & -12 & 17 & -4 & 2 & 2 & 2 & - \\
\hline Dorsal striatum (R) & 13 & 17 & -5 & 2 & 2 & 2 & - \\
\hline Fusiform gyrus (L) & -33 & -37 & -16 & 1 & 3 & 3 & Choice \\
\hline Fusiform gyrus (R) & 27 & -60 & -8 & 3 & 4 & 4 & \\
\hline Hippocampus (L) & -26 & -12 & -22 & 1 & 1 & 1 & \\
\hline Hippocampus (R) & 25 & -11 & -23 & 1 & 1 & & - \\
\hline Insula (L) & -36 & 2 & 4 & 5 & 5 & & - \\
\hline Insula (R) & 38 & 10 & 4 & 5 & 5 & 5 & - \\
\hline Intracalcarine cortex (L) & -8 & -79 & 7 & 3 & 4 & 4 & Choice \\
\hline Intracalcarine cortex $(R)$ & 5 & -78 & 7 & & 4 & 4 & Choice \\
\hline Lateral OFC (L) & -30 & 20 & -20 & & 4 & 4 & - \\
\hline Lateral OFC (R) & 30 & 20 & -18 & 4 & 4 & 4 & - \\
\hline Medial OFC (L) & -14 & 19 & & 3 & 3 & 3 & - \\
\hline Medial OFC (R) & 14 & & -17 & 3 & 3 & 3 & - \\
\hline Pre/postcentral gyrus (L) & -42 & -17 & 53 & 2 & 2 & 2 & - \\
\hline Pre/postcentral gyrus (R) & 40 & -20 & 53 & 2 & 2 & 2 & - \\
\hline Precuneus (L) & -7 & -59 & 26 & 6 & 6 & 6 & - \\
\hline Precuneus (R) & 13 & -62 & 28 & 6 & 6 & 6 & - \\
\hline Thalamus (L) & -14 & -20 & 0 & 5 & 5 & 5 & - \\
\hline Thalamus $(\mathrm{R})$ & 14 & -20 & 0 & 5 & 5 & 5 & - \\
\hline Ventral striatum (L) & -10 & 17 & 7 & 7 & 7 & 7 & - \\
\hline Ventral striatum (R) & 10 & 16 & 7 & 7 & 7 & 7 & - \\
\hline Ventrolateral PFC (L) & -42 & 46 & -1 & 2 & 8 & 2 & Sweet \\
\hline Ventrolateral PFC (R) & 43 & 48 & -6 & 2 & 8 & 2 & Sweet \\
\hline Ventromedial PFC (L) & -5 & 43 & -8 & 2 & 6 & 2 & Sweet \\
\hline Ventromedial PFC (R) & 6 & 42 & -4 & 2 & 6 & 2 & Sweet \\
\hline
\end{tabular}


Figure 5: Differences in Community Structure and Metrics associated with BMl and Posttest Performance.

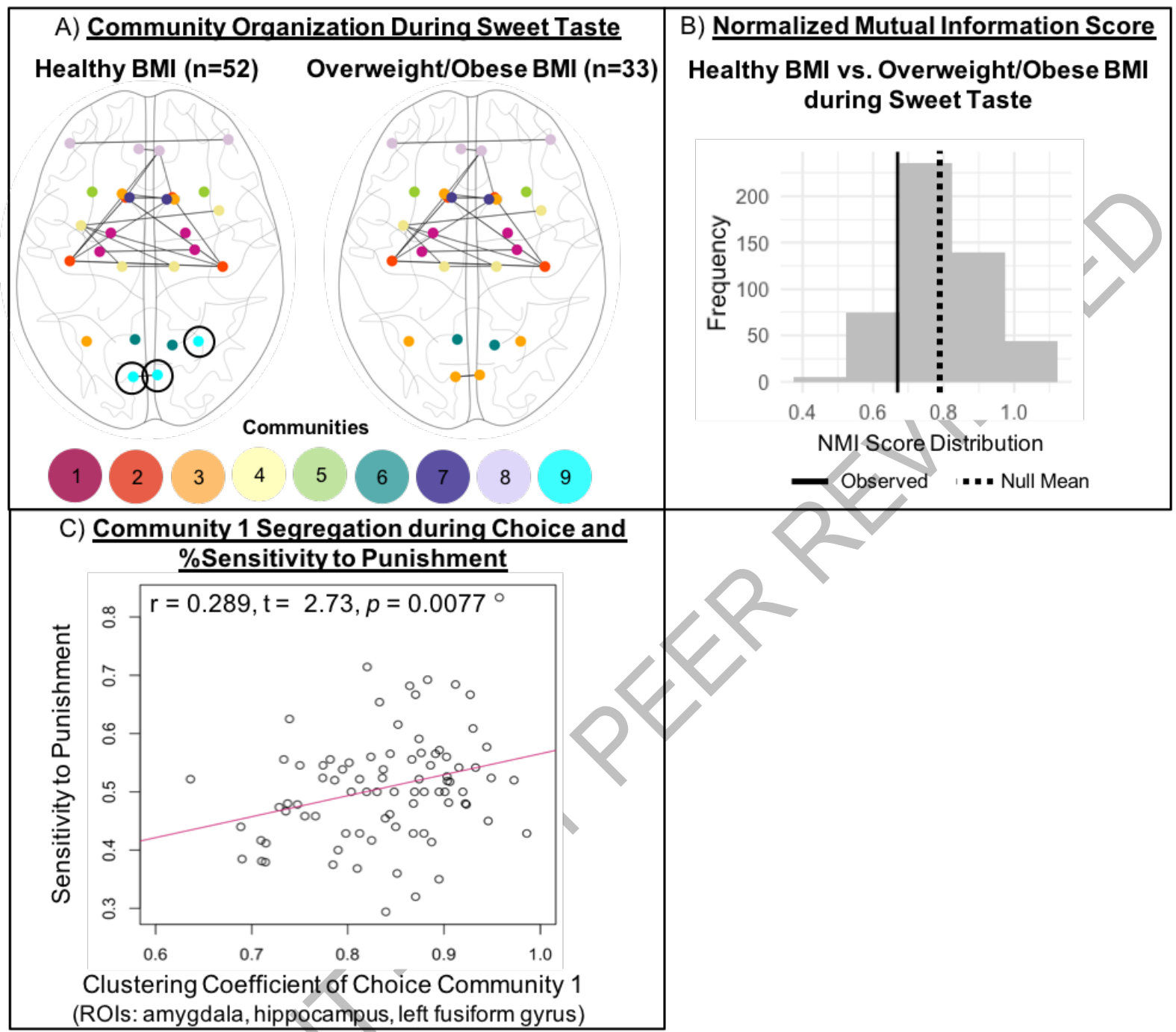

\section{DISCUSSION}

\section{Summary}

Reinforcement learning via food reward and punishment is the foundation of dietary choice, yet few studies have examined how the brain responds to taste reinforcers using a responsedependent task. Here, 85 healthy, young adults completed an instrumental conditioning task [42] to assess whole-brain response and network organization during choice and reinforcement via sweet tastes and bitter tastes. Our data suggest that choice and reinforcement via reward and punishment are associated with distinct patterns of brain response and network organization. Further, we found that BMI was associated with differential network organization of 
regions in the visual cortex, and that clustering of a community involved in learning and memory during choice was related to improved avoidance of a cue associated with bitter taste. Together, these results indicate that measures of brain network structure and topology are associated with individual differences in weight status and ability to learn from taste.

\section{Appetitive PST Performance}

Diverging from previous iterations of the probabilistic selection task [42,69], performance on the appetitive probabilistic selection task was close to chance in our sample. A major difference between the appetitive PST and the original version of the task is the use of sweet and bitter tasting beverages as feedback. Taste is a primary reinforcer, but it has challenges in research application because the motivational salience of foods change with satiation [70] and habituation to tastes [71]. Satiation, especially the development of sensory specific satiety, can decrease the motivational value of foods over time [72,73]. Further, habituation decreases operant response to rewards and punishment over exposures [71,74]. Changes to motivation over the course of the appetitive PST could contribute to the divergence of the present posttest performance from prior studies using secondary outcomes. Further, the possibility of habituation to the bitter taste or even the development of preference for the bitter taste may explain our results. Exposure increases liking of foods [75] and repeated exposure enhances the hedonic evaluation of a bitter-flavored beverage [76]. Together, sensory specific satiety and habituation may change the reward and punishment associated with sweet and bitter tastes, narrowing the difference in motivational salience between the two flavors over the course of training. In the present data posttest scores below chance $(50 \%$ choose $A$ and avoid $B$ ) could indicate the formation of preference for shapes associated with the bitter taste. Hedonic valuation of the stimuli was not assessed at the end of the task, so we are unable to test this interpretation. Notably, in prior studies with non-taste outcomes, posttest performance on the PST was decreased in participants with obesity [69]. In the present sample, posttest performance was weakly negatively related to BMI, but the effect was not statistically significant. Thus, confounding effects of habituation/satiation on posttest performance may have obscured the relationship between $\mathrm{BMI}$ and sensitivity to outcomes in our sample.

\section{Brain Response and Network Organization during Appetitive PST}

During the appetitive PST training, we observed robust patterns of brain response across all conditions. During the choice period, including a button press in response to the question "which [shape] is correct', BOLD response was found in regions relating to these systems. BOLD 
response in the visual cortex, precentral gyrus, posterior temporal lobe, hippocampus and caudate, may relate to visual processing, motor response, language processing [77], working memory [78,79], and reinforcer expectations respectively [80,81]. While not all hypothesized regions showed response during choice, the observed response fits within the current model of instrumental-response in the brain [82]. Extant research on brain response to taste shows patterns of response to palatable (high sugar and high fat) tastes consistent with brain response to the sweet, reward beverage found here $[27,83,84]$. When reward was compared to punishment, we observed greater BOLD response in the precuneus, oral somatosensory cortex, and OFC, regions that all show response to palatable tastes compared to a neutral solution [85]. Additionally, greater response in caudate was observed, similar to other studies of palatable taste [86-88]. These results add further evidence to support brain response to palatable taste in the describe regions. When compared to reward, punishment was associated with greater response in the dorsal anterior cingulate cortex (dACC). This result was unexpected, as we hypothesized that punishment would be related to BOLD response in the OFC and amygdala. The dACC is thought to connect midbrain motivational areas to prefrontal control regions [89], and acts as a 'controller', integral for reward based decision making [90,91] and adaptation [92]. Given its role in updating response, dACC response during punishment may reflect an update to expectations following the bitter taste.

Network organization was examined to further characterize response to the task during choice, sweet taste administration, and bitter taste administration. The brain is a complex system, and network approaches provide unique information about the coordination of brain response to a stimulus and the function of different communities, or clusters of ROls [35]. Community structure reflects functional specialization [35], and the organization (or member assignments) of communities shed light on the subprocesses involved in cognition [93]. Across all phases of the task, we observed highly dimensional brain networks, with eight, nine, and eight communities identified during choice, sweet taste, and bitter taste, respectively. Community assignment for about $70 \%$ of the regions examined was conserved in all conditions of the task. Unique community assignment was identified for ROls in the visual cortex and orbitofrontal cortex during choice. In this condition, ROls in the right fusiform gyrus, intracalcarine cortex, and medial OFC organize into the same community. The medial OFC monitors stimulus-reinforcer relationships [94]. Its connectivity with visual regions during choice may represent the incorporation of stimuli perception with reinforcer value assessment, that could underpin decisions between cues. Unique community assignment was found for ROls in the ventromedial and ventrolateral prefrontal cortex during response to the sweet taste. During 
bitter taste, the vmPFC and VIPFC were grouped into a larger community with the dorsal striatum and pre/postcentral gyrus ROIs, reflecting connectivity the cortico-striatal loop that is critical for feedback-based learning and action updating [95,96]. Conversely, during sweet taste, this cortico-striatal community reorganized, such that the vmPFC was grouped with the precuneus, while the vIPFC was assigned to its own community. The vmPFC encodes the value of choices and outcomes [20]. The function of the precuneus is multifaceted, its response is related to self-consciousness, pain, and memory [97]. The vmPFC and precuneus are functionally connected at rest $[97,98]$, and BOLD response in both regions increases with certainty in a choice between a desired food image and an undesired food image [99]. Increased connectivity of the vmPFC and precuneus during sweet taste may reflect the positive feedback conveyed by taste. Further, the vIPFC, was organized into its own community assignment during sweet taste, Dissociation of the vIPFC, which is involved in behavioral regulation [96] and learning stimulus-response relationships [100], from the vmPFC and striatum may reflect differences in the coordination of response to a food reward versus a food punishment.

In consideration of the wide variance in task performance between individuals, we tested for differences in network organization and topology associated with task performance. While we did not see differences in network organization between participants who performed above chance versus below chance on the appetitive PST posttest, we found that increasing segregation of the amygdala, hippocampus, and right fusiform gyrus community during choice was significantly associated with increased sensitivity to punishment, as measured by the PST posttest. This community represents memory and learning regions $[101,102]$, and connectivity of the amygdala and hippocampus predicts behavioral adaptation to positive and negative feedback [101]. Increasing segregation of this community reflects stronger within-community connectivity and dissociation from the rest of the network [103], and allows for more efficient specialization of communities. Higher segregation may reflect more efficient communication between the areas involved in learning, memory, and visual response. This in turn, could underpin increased sensitivity to food punishment observed on the posttest.

\section{Effects of BMI on Task Performance and Network Organization}

One factor demonstrated to affect PST task performance is obesity. Individuals with obesity show insensitivity to negative outcomes on the PST task [69], and more broadly obesity is related to insensitivity to negative prediction errors and punishment $[104,105]$. However, in the present sample we did not find significant associations between BMI and posttest performance. 
When network organization was compared between BMI groups, we observed differences in network organization during sweet taste between healthy BMI and overweight/obese BMI groups in bilateral intracalcarine cortex ROls and the right fusiform gyrus, where these ROls were integrated into a broader community to the left fusiform gyrus and medial OFC in the overweight/obese group. Thus, during sweet taste, network organization among overweight/obese participants reflect increased connectivity of visual ROls with the orbitofrontal cortex during sweet taste. Similarly, Geha et al reported increased global connectivity of the visual cortex in obesity during consumption of a palatable milkshake [106]. Further, obesity is associated with stronger visual cortex response to food images [107]. Our results also support the interpretation that elevated BMI may be related to greater orientation to external stimuli during consumption [106], which could contribute to excessive intake.

\section{Limitations}

This study has notable strengths and limitations. While our study is the first to adapt the Probabilistic Selection Task to use a primary taste reinforcer, we were not able to reliably identify learning in our sample. Because of this, we were not able to account for learning and expectancy in our analyses of BOLD response and network structure during training. It is possible that response, especially that of midbrain ROls that respond strongly to prediction error $[17,108]$ may be misattributed to response to reward or punishment. Within individuals, this may not impact overall results because prediction error should be distributed across reward and punishment evenly, but across individuals this effect may be meaningful, since feedback was randomized, and participants received different numbers of prediction error trials (e.g. choose correct shape, but received bitter taste). While the rate of prediction error trials was not correlated with posttest accuracy, it may have contributed to differences in trial-by-trial brain response. Future studies of brain response during taste-mediated instrumental conditioning should aim to increase the number of taste events or include additional training to improve performance and allow for a computational model of reinforcement learning to be fit to behavioral data. Second, graph-theory based network analyses provide unique information to further characterize brain response during choice, sweet taste administration, and bitter taste administration. However, these types of analyses limit the scope of brain regions examined. While the ROls selected were drawn from research on reinforcement learning and taste administration, it is possible that regions meaningful to network structure were not included in our analytic set. With more trials, future studies may be able to identify other regions important to network structure during choice, reward, and punishment. Similarly, we did not observe any 
meaningful differences between participants who performed above chance on the posttest and those who performed below chance. Some unmeasured construct may relate to individual differences in posttest performance, such as impulsivity [109] or episodic memory [110]. Finally, this study did not collect hedonic ratings of the task stimuli following completion of the PST training. Without this data we are not able to test for changes in the motivational salience of the sweet taste and bitter taste following training, limiting our ability to address the possible effects of satiation or habituation to the taste on posttest performance.

\section{Conclusions}

How we learn from taste reinforcement is an important driver of food choices, and provides a window into how food reward may motivate individuals to choose palatable foods. Our results are the first to examine network modularity during completion of an instrumental conditioning task with response-dependent taste administration. We found that brain networks during choice, sweet taste and bitter taste are similar with key differences in visual cortex areas and prefrontal regions important for value representation and behavioral regulation. We found that during sweet taste, participants with overweight/obese BMI showed broader connectivity of visual cortex regions than participants with healthy BMI, suggesting that elevated BMI is related to greater integration of external stimuli during consumption. Also, segregation of a community involved in learning and memory was associated with improved posttest performance, indicating that efficient processing within this community is associated with an improved ability to avoid bitter tastes. Together, results shed light on the complex interaction of brain regions responding to taste-based reinforcement learning, support that individual differences in connectivity of visual regions and segregation of learning and memory areas are related to $\mathrm{BMI}$ and learning from taste. 


\section{Acknowledgements}

The authors would like to thank the participants who took part in the study. The authors would also like to thank the Neuropsychology of Ingestive Behavior lab, specifically Katie Gandee, Lia Bauert, Peter Dihn, Ryesa Mansoor, Megan Neff, and Brian Brown for assistance in data collection and study administration.

S.Q.P. was supported by the German Research Foundation Grants PA 2682/1-1, PA 2682/2-1 and by a grant from the German Ministry of Education and Research (BMBF) and the State of Brandenburg (DZD grant FKZ 82DZD00302). 


\section{Bibliography}

[1] C.L. Ogden, M.D. Carroll, B.K. Kit, K.M. Flegal, Prevalence of obesity and trends in body mass index among US children and adolescents, 1999-2010., JAMA. 307 (2012) 483490. doi:10.1001/jama.2012.40.

[2] C. Weyer, T. Funahashi, S. Tanaka, K. Hotta, Y. Matsuzawa, R.E. Pratley, et al., Hypoadiponectinemia in obesity and type 2 diabetes: close association with insulin resistance and hyperinsulinemia., J. Clin. Endocrinol. Metab. 86 (2001) 1930-1935. doi:10.1210/jcem.86.5.7463.

[3] I. Shai, R. Jiang, J.E. Manson, M.J. Stampfer, W.C. Willett, G.A. Colditz, et al., Ethnicity, obesity, and risk of type 2 diabetes in women: a 20 -year follow-up study., Diabetes Care. 29 (2006) 1585-1590. doi:10.2337/dc06-0057.

[4] S.E. Kahn, R.L. Hull, K.M. Utzschneider, Mechanisms linking obesity to insulin resistance and type 2 diabetes., Nature. 444 (2006) 840-846. doi:10.1038/nature05482.

[5] J. Font-Burgada, B. Sun, M. Karin, Obesity and Cancer: The Oil that Feeds the Flame., Cell Metab. 23 (2016) 48-62. doi:10.1016/j.cmet.2015.12.015.

[6] A.G. Renehan, M. Tyson, M. Egger, R.F. Heller, M. Zwahlen, Body-mass index and incidence of cancer: a systematic review and meta-analysis of prospective observational studies., Lancet. 371 (2008) 569-578. doi:10.1016/S0140-6736(08)60269-X.

[7] H.B. Hubert, M. Feinleib, P.M. McNamara, W.P. Castelli, Obesity as an independent risk factor for cardiovascular disease: a 26-year follow-up of participants in the Framingham Heart Study., Circulation. 67 (1983) 968-977. doi:10.1161/01.cir.67.5.968.

[8] J.R. Sowers, Obesity as a cardiovascular risk factor., Am. J. Med. 115 Suppl 8A (2003) 37S-41S. doi:10.1016/j.amjmed.2003.08.012.

[9] S.M. Attard, A.H. Herring, A.G. Howard, P. Gordon-Larsen, Longitudinal trajectories of $\mathrm{BMI}$ and cardiovascular disease risk: the national longitudinal study of adolescent health., Obesity (Silver Spring). 21 (2013) 2180-2188. doi:10.1002/oby.20569.

[10] G. Egger, J. Dixon, Beyond obesity and lifestyle: a review of 21st century chronic disease determinants., Biomed Res. Int. 2014 (2014) 731685. doi:10.1155/2014/731685.

[11] D.J. Mela, Determinants of food choice: relationships with obesity and weight control., Obes Res. 9 Suppl 4 (2001) 249S-255S. doi:10.1038/oby.2001.127.

[12] A. Drewnowski, Taste preferences and food intake., Annu. Rev. Nutr. 17 (1997) 237253. doi:10.1146/annurev.nutr.17.1.237.

[13] F. Johnson, M. Pratt, J. Wardle, Dietary restraint and self-regulation in eating behavior., Int. J. Obes. 36 (2012) 665-674. doi:10.1038/ijo.2011.156.

[14] M.C. Nelson, P. Gordon-Larsen, K.E. North, L.S. Adair, Body mass index gain, fast food, and physical activity: effects of shared environments over time., Obesity (Silver Spring). 14 (2006) 701-709. doi:10.1038/oby.2006.80.

[15] E.J. Boyland, S. Nolan, B. Kelly, C. Tudur-Smith, A. Jones, J.C. Halford, et al., Advertising as a cue to consume: a systematic review and meta-analysis of the effects of acute exposure to unhealthy food and nonalcoholic beverage advertising on intake in children and adults., Am. J. Clin. Nutr. 103 (2016) 519-533. doi:10.3945/ajcn.115.120022.

[16] A. Rangel, Regulation of dietary choice by the decision-making circuitry., Nat. Neurosci. 
16 (2013) 1717-1724. doi:10.1038/nn.3561.

[17] T.A. Hare, J. O'Doherty, C.F. Camerer, W. Schultz, A. Rangel, Dissociating the role of the orbitofrontal cortex and the striatum in the computation of goal values and prediction errors., J. Neurosci. 28 (2008) 5623-5630. doi:10.1523/JNEUROSCI.1309-08.2008.

[18] M.F.S. Rushworth, T.E.J. Behrens, Choice, uncertainty and value in prefrontal and cingulate cortex., Nat. Neurosci. 11 (2008) 389-397. doi:10.1038/nn2066.

[19] V.S. Chib, A. Rangel, S. Shimojo, J.P. O'Doherty, Evidence for a common representation of decision values for dissimilar goods in human ventromedial prefrontal cortex., J. Neurosci. 29 (2009) 12315-12320. doi:10.1523/JNEUROSCI.2575-09.2009.

[20] A. Rangel, T. Hare, Neural computations associated with goal-directed choice., Curr. Opin. Neurobiol. 20 (2010) 262-270. doi:10.1016/j.conb.2010.03.001.

[21] Y.Q. Low, K. Lacy, R. Keast, The role of sweet taste in satiation and satiety., Nutrients. 6 (2014) 3431-3450. doi:10.3390/nu6093431.

[22] J.A. Mennella, M.Y. Pepino, D.R. Reed, Genetic and environmental determinants of bitter perception and sweet preferences., Pediatrics. 115 (2005) e216-22. doi:10.1542/peds.2004-1582.

[23] L.H. Epstein, J.J. Leddy, J.L. Temple, M.S. Faith, Food reinforcement and eating: a multilevel analysis., Psychol. Bull. 133 (2007) 884-906. doi:10.1037/00332909.133.5.884.

[24] J.P. O’Doherty, J. Cockburn, W.M. Pauli, Learning, reward, and decision making., Annu. Rev. Psychol. 68 (2017) 73-100. doi:10.1146/annurev-psych-010416-044216.

[25] C. Kure Liu, P.V. Joseph, D.E. Feldman, D.S. Kroll, J.A. Burns, P. Manza, et al., Brain imaging of taste perception in obesity: a review., Curr. Nutr. Rep. 8 (2019) 108-119. doi:10.1007/s13668-019-0269-y.

[26] E. Stice, K. Burger, Neural vulnerability factors for obesity., Clin. Psychol. Rev. 68 (2019) 38-53. doi:10.1016/j.cpr.2018.12.002.

[27] J. O'Doherty, E.T. Rolls, S. Francis, R. Bowtell, F. McGlone, Representation of pleasant and aversive taste in the human brain., J. Neurophysiol. 85 (2001) 1315-1321. doi:10.1152/jn.2001.85.3.1315.

[28] D.H. Zald, M.C. Hagen, J.V. Pardo, Neural correlates of tasting concentrated quinine and sugar solutions., J. Neurophysiol. 87 (2002) 1068-1075. doi:10.1152/jn.00358.2001.

[29] A.M. Aertsen, G.L. Gerstein, M.K. Habib, G. Palm, Dynamics of neuronal firing correlation: modulation of "effective connectivity"., J. Neurophysiol. 61 (1989) 900-917. doi:10.1152/jn.1989.61.5.900.

[30] E. Camara, A. Rodriguez-Fornells, T.F. Münte, Functional connectivity of reward processing in the brain., Front. Hum. Neurosci. 2 (2008) 19. doi:10.3389/neuro.09.019.2008.

[31] D.M. Small, Taste representation in the human insula., Brain Struct. Funct. 214 (2010) 551-561. doi:10.1007/s00429-010-0266-9.

[32] H.-J. Park, K. Friston, Structural and functional brain networks: from connections to cognition., Science. 342 (2013) 1238411. doi:10.1126/science.1238411.

[33] J.R. Cohen, M. D'Esposito, The segregation and integration of distinct brain networks and their relationship to cognition., J. Neurosci. 36 (2016) 12083-12094. doi:10.1523/JNEUROSCI.2965-15.2016. 
[34] D.S. Bassett, O. Sporns, Network neuroscience., Nat. Neurosci. 20 (2017) 353-364. doi:10.1038/nn.4502.

[35] O. Sporns, R.F. Betzel, Modular Brain Networks., Annu. Rev. Psychol. 67 (2016) 613640. doi:10.1146/annurev-psych-122414-033634.

[36] R.T. Gerraty, J.Y. Davidow, K. Foerde, A. Galvan, D.S. Bassett, D. Shohamy, Dynamic Flexibility in Striatal-Cortical Circuits Supports Reinforcement Learning., J. Neurosci. 38 (2018) 2442-2453. doi:10.1523/JNEUROSCI.2084-17.2018.

[37] D.S. Bassett, N.F. Wymbs, M.A. Porter, P.J. Mucha, J.M. Carlson, S.T. Grafton, Dynamic reconfiguration of human brain networks during learning., Proc. Natl. Acad. Sci. USA. 108 (2011) 7641-7646. doi:10.1073/pnas.1018985108.

[38] K.-I. Amemori, L.G. Gibb, A.M. Graybiel, Shifting responsibly: the importance of striatal modularity to reinforcement learning in uncertain environments., Front. Hum. Neurosci. 5 (2011) 47. doi:10.3389/fnhum.2011.00047.

[39] M.R. Delgado, Reward-related responses in the human striatum., Ann. N. Y. Acad. Sci. 1104 (2007) 70-88. doi:10.1196/annals.1390.002.

[40] J. Jensen, H. Walter, Incentive motivational salience and the human brain., Restor Neurol Neurosci. 32 (2014) 141-147. doi:10.3233/RNN-139006.

[41] S.M. Beck, H.S. Locke, A.C. Savine, K. Jimura, T.S. Braver, Primary and secondary rewards differentially modulate neural activity dynamics during working memory., PLoS One. 5 (2010) e9251. doi:10.1371/journal.pone.0009251.

[42] M.J. Frank, L.C. Seeberger, R.C. O'reilly, By carrot or by stick: cognitive reinforcement learning in parkinsonism., Science. 306 (2004) 1940-1943. doi:10.1126/science.1102941.

[43] G. Charalambous, Chemistry of foods and beverages: recent developments, Elsevier, 2012.

[44] J.W. Peirce, PsychoPy--Psychophysics software in Python., J. Neurosci. Methods. 162 (2007) 8-13. doi:10.1016/j.jneumeth.2006.11.017.

[45] O. Esteban, C. Markiewicz, R.W. Blair, C. Moodie, A.I. Isik, A. Erramuzpe Aliaga, et al., FMRIPrep: a robust preprocessing pipeline for functional MRI, BioRxiv. (2018). doi:10.1101/306951.

[46] K.J. Gorgolewski, T. Auer, V.D. Calhoun, R.C. Craddock, S. Das, E.P. Duff, et al., The brain imaging data structure, a format for organizing and describing outputs of neuroimaging experiments., Sci. Data. 3 (2016) 160044. doi:10.1038/sdata.2016.44.

[47] M.W. Woolrich, T.E.J. Behrens, C.F. Beckmann, M. Jenkinson, S.M. Smith, Multilevel linear modelling for FMRI group analysis using Bayesian inference., Neuroimage. 21 (2004) 1732-1747. doi:10.1016/j.neuroimage.2003.12.023.

[48] M.W. Woolrich, B.D. Ripley, M. Brady, S.M. Smith, Temporal autocorrelation in univariate linear modeling of FMRI data., Neuroimage. 14 (2001) 1370-1386. doi:10.1006/nimg.2001.0931.

[49] S.M. Smith, T.E. Nichols, Threshold-free cluster enhancement: addressing problems of smoothing, threshold dependence and localisation in cluster inference., Neuroimage. 44 (2009) 83-98. doi:10.1016/j.neuroimage.2008.03.061.

[50] J. Rissman, A. Gazzaley, M. D’Esposito, Measuring functional connectivity during distinct stages of a cognitive task., Neuroimage. 23 (2004) 752-763. 
doi:10.1016/j.neuroimage.2004.06.035.

[51] J.A. Mumford, B.O. Turner, F.G. Ashby, R.A. Poldrack, Deconvolving BOLD activation in event-related designs for multivoxel pattern classification analyses., Neuroimage. 59 (2012) 2636-2643. doi:10.1016/j.neuroimage.2011.08.076.

[52] J.D. Power, A.L. Cohen, S.M. Nelson, G.S. Wig, K.A. Barnes, J.A. Church, et al., Functional network organization of the human brain., Neuron. 72 (2011) 665-678. doi:10.1016/j.neuron.2011.09.006.

[53] B.A. Seitzman, C. Gratton, S. Marek, R.V. Raut, N.U. Dosenbach, B.L. Schlaggar, et al., A set of functionally-defined brain regions with improved representation of the subcortex and cerebellum, BioRxiv. (2018). doi:10.1101/450452.

[54] L.N. van der Laan, D.T.D. de Ridder, M.A. Viergever, P.A.M. Smeets, The first taste is always with the eyes: a meta-analysis on the neural correlates of processing visual food cues., Neuroimage. 55 (2011) 296-303. doi:10.1016/j.neuroimage.2010.11.055.

[55] A.W.K. Yeung, T.K. Goto, W.K. Leung, Basic taste processing recruits bilateral anteroventral and middle dorsal insulae: An activation likelihood estimation metaanalysis of fMRI studies., Brain Behav. 7 (2017) e00655. doi:10.1002/brb3.655.

[56] I.C. Ballard, V.P. Murty, R.M. Carter, J.J. Maclnnes, S.A. Huettel, R.A. Adcock, Dorsolateral prefrontal cortex drives mesolimbic dopaminergic regions to initiate motivated behavior., J. Neurosci. 31 (2011) 10340-10346. doi:10.1523/JNEUROSCI.0895-11.2011.

[57] S.H. Kim, H. Yoon, H. Kim, S. Hamann, Individual differences in sensitivity to reward and punishment and neural activity during reward and avoidance learning., Soc. Cogn. Affect. Neurosci. 10 (2015) 1219-1227. doi:10.1093/scan/nsv007.

[58] D.M. Small, Flavor is in the brain., Physiol. Behav. 107 (2012) 540-552. doi:10.1016/j.physbeh.2012.04.011.

[59] M. Rubinov, O. Sporns, Weight-conserving characterization of complex functional brain networks., Neuroimage. 56 (2011) 2068-2079. doi:10.1016/j.neuroimage.2011.03.069.

[60] A.A. Hagberg, D.A. Schult, P.J. Swart, Exploring Network Structure, Dynamics, and Function using NetworkX, (2008).

[61] T. Kluyver, B. Ragan-Kelley, F. Pérez, B.E. Granger, M. Bussonnier, J. Frederic, et al., Jupyter Notebooks-a publishing format for reproducible computational workflows., ELPUB. (2016) 87.

[62] V.D. Blondel, J.-L. Guillaume, R. Lambiotte, E. Lefebvre, Fast unfolding of communities in large networks, J. Stat. Mech. 2008 (2008) P10008. doi:10.1088/17425468/2008/10/P10008.

[63] J. Saramäki, M. Kivelä, J.-P. Onnela, K. Kaski, J. Kertész, Generalizations of the clustering coefficient to weighted complex networks., Phys. Rev. E, Stat. Nonlin. Soft. Matter. Phys. 75 (2007) 027105. doi:10.1103/PhysRevE.75.027105.

[64] U. Brandes, On variants of shortest-path betweenness centrality and their generic computation, Soc. Networks. 30 (2008) 136-145. doi:10.1016/j.socnet.2007.11.001.

[65] M. Rubinov, O. Sporns, Complex network measures of brain connectivity: uses and interpretations., Neuroimage. 52 (2010) 1059-1069. doi:10.1016/j.neuroimage.2009.10.003.

[66] A.F. McDaid, D. Greene, N. Hurley, Normalized mutual information to evaluate 
overlapping community finding algorithms., ArXiv Preprint ArXiv:1110.2515. (2011).

[67] S. Marek, K. Hwang, W. Foran, M.N. Hallquist, B. Luna, The contribution of network organization and integration to the development of cognitive control., PLoS Biol. 13 (2015) e1002328. doi:10.1371/journal.pbio.1002328.

[68] D. Bates, M. Mächler, B. Bolker, S. Walker, Fitting linear mixed-effects models using Ime4., ArXiv Preprint ArXiv:1406.5823. (2014).

[69] G. Coppin, S. Nolan-Poupart, M. Jones-Gotman, D.M. Small, Working memory and reward association learning impairments in obesity., Neuropsychologia. 65 (2014) 146155. doi:10.1016/j.neuropsychologia.2014.10.004.

[70] B.J. Rolls, E.T. Rolls, E.A. Rowe, K. Sweeney, Sensory specific satiety in man., Physiol. Behav. 27 (1981) 137-142. doi:10.1016/0031-9384(81)90310-3.

[71] J.L. Temple, A.M. Giacomelli, J.N. Roemmich, L.H. Epstein, Habituation and withinsession changes in motivated responding for food in children., Appetite. 50 (2008) 390396. doi:10.1016/j.appet.2007.09.005.

[72] R.C. Havermans, T. Janssen, J.C.A.H. Giesen, A. Roefs, A. Jansen, Food liking, food wanting, and sensory-specific satiety., Appetite. 52 (2009) 222-225. doi:10.1016/j.appet.2008.09.020.

[73] A. Sclafani, K. Ackroff, The relationship between food reward and satiation revisited., Physiol. Behav. 82 (2004) 89-95. doi:10.1016/j.physbeh.2004.04.045.

[74] F.K. Mcsweeney, J.M. Roll, Do animals satiate or habituate to repeatedly presented reinforcers?, Psychon. Bull. Rev. 5 (1998) 428-442. doi:10.3758/BF03208818.

[75] P. Pliner, The effects of mere exposure on liking for edible substances., Appetite. 3 (1982) 283-290. doi:10.1016/s0195-6663(82)80026-3.

[76] L.J. Stein, H. Nagai, M. Nakagawa, G.K. Beauchamp, Effects of repeated exposure and health-related information on hedonic evaluation and acceptance of a bitter beverage., Appetite. 40 (2003) 119-129. doi:10.1016/s0195-6663(02)00173-3.

[77] G. Spitsyna, J.E. Warren, S.K. Scott, F.E. Turkheimer, R.J.S. Wise, Converging language streams in the human temporal lobe., J. Neurosci. 26 (2006) 7328-7336. doi:10.1523/JNEUROSCI.0559-06.2006.

[78] A. Ben-Yakov, Y. Dudai, Constructing realistic engrams: poststimulus activity of hippocampus and dorsal striatum predicts subsequent episodic memory., J. Neurosci. 31 (2011) 9032-9042. doi:10.1523/JNEUROSCI.0702-11.2011.

[79] C. Ranganath, M.X. Cohen, C. Dam, M. D’Esposito, Inferior temporal, prefrontal, and hippocampal contributions to visual working memory maintenance and associative memory retrieval., J. Neurosci. 24 (2004) 3917-3925. doi:10.1523/JNEUROSCI.505303.2004.

[80] W. Schultz, Neuronal reward and decision signals: from theories to data., Physiol. Rev. 95 (2015) 853-951. doi:10.1152/physrev.00023.2014.

[81] W.M. Pauli, G. Gentile, S. Collette, J.M. Tyszka, J.P. O'Doherty, Evidence for modelbased encoding of Pavlovian contingencies in the human brain., Nat. Commun. 10 (2019) 1099. doi:10.1038/s41467-019-08922-7.

[82] R.J. Dolan, P. Dayan, Goals and habits in the brain., Neuron. 80 (2013) 312-325. doi:10.1016/j.neuron.2013.09.007.

[83] E. Stice, S. Spoor, C. Bohon, M.G. Veldhuizen, D.M. Small, Relation of reward from 
food intake and anticipated food intake to obesity: a functional magnetic resonance imaging study., J. Abnorm. Psychol. 117 (2008) 924-935. doi:10.1037/a0013600.

[84] J.P. O'Doherty, R. Deichmann, H.D. Critchley, R.J. Dolan, Neural responses during anticipation of a primary taste reward., Neuron. 33 (2002) 815-826. doi:10.1016/s08966273(02)00603-7.

[85] E.T. Rolls, Sensory processing in the brain related to the control of food intake., Proc Nutr Soc. 66 (2007) 96-112. doi:10.1017/S0029665107005332.

[86] E. Stice, K.S. Burger, S. Yokum, Relative ability of fat and sugar tastes to activate reward, gustatory, and somatosensory regions., Am. J. Clin. Nutr. 98 (2013) 1377-1384. doi:10.3945/ajcn.113.069443.

[87] M. Alonso-Alonso, S.C. Woods, M. Pelchat, P.S. Grigson, E. Stice, S. Farooqi, et al., Food reward system: current perspectives and future research needs., Nutr. Rev. 73 (2015) 296-307. doi:10.1093/nutrit/nuv002.

[88] G.E. Shearrer, E. Stice, K.S. Burger, Adolescents at high risk of obesity show greater striatal response to increased sugar content in milkshakes., Am. J. Clin. Nutr. 107 (2018) 859-866. doi:10.1093/ajcn/nqy050.

[89] M. Beckmann, H. Johansen-Berg, M.F.S. Rushworth, Connectivity-based parcellation of human cingulate cortex and its relation to functional specialization., J. Neurosci. 29 (2009) 1175-1190. doi:10.1523/JNEUROSCI.3328-08.2009.

[90] Z.M. Williams, G. Bush, S.L. Rauch, G.R. Cosgrove, E.N. Eskandar, Human anterior cingulate neurons and the integration of monetary reward with motor responses., Nat. Neurosci. 7 (2004) 1370-1375. doi:10.1038/nn1354.

[91] A.A. Marsh, K.S. Blair, M. Vythilingam, S. Busis, R.J.R. Blair, Response options and expectations of reward in decision-making: the differential roles of dorsal and rostral anterior cingulate cortex., Neuroimage. 35 (2007) 979-988. doi:10.1016/j.neuroimage.2006.11.044.

[92] S.A. Sheth, M.K. Mian, S.R. Patel, W.F. Asaad, Z.M. Williams, D.D. Dougherty, et al., Human dorsal anterior cingulate cortex neurons mediate ongoing behavioural adaptation., Nature. 488 (2012) 218-221. doi:10.1038/nature11239.

[93] G.S. Wig, Segregated systems of human brain networks., Trends Cogn. Sci. (Regul. Ed.). 21 (2017) 981-996. doi:10.1016/j.tics.2017.09.006.

[94] R. Elliott, R.J. Dolan, C.D. Frith, Dissociable functions in the medial and lateral orbitofrontal cortex: evidence from human neuroimaging studies., Cereb. Cortex. 10 (2000) 308-317. doi:10.1093/cercor/10.3.308.

[95] D. Shohamy, C.E. Myers, S. Grossman, J. Sage, M.A. Gluck, R.A. Poldrack, Corticostriatal contributions to feedback-based learning: converging data from neuroimaging and neuropsychology., Brain. 127 (2004) 851-859. doi:10.1093/brain/awh100.

[96] B.J. Levy, A.D. Wagner, Cognitive control and right ventrolateral prefrontal cortex: reflexive reorienting, motor inhibition, and action updating., Ann. N. Y. Acad. Sci. 1224 (2011) 40-62. doi:10.1111/j.1749-6632.2011.05958.x.

[97] A.E. Cavanna, M.R. Trimble, The precuneus: a review of its functional anatomy and behavioural correlates., Brain. 129 (2006) 564-583. doi:10.1093/brain/awl004.

[98] M.D. Greicius, B. Krasnow, A.L. Reiss, V. Menon, Functional connectivity in the resting brain: a network analysis of the default mode hypothesis., Proc. Natl. Acad. Sci. USA. 
100 (2003) 253-258. doi:10.1073/pnas.0135058100.

[99] B. De Martino, S.M. Fleming, N. Garrett, R.J. Dolan, Confidence in value-based choice., Nat. Neurosci. 16 (2013) 105-110. doi:10.1038/nn.3279.

[100] C.A. Boettiger, M. D'Esposito, Frontal networks for learning and executing arbitrary stimulus-response associations., J. Neurosci. 25 (2005) 2723-2732. doi:10.1523/JNEUROSCI.3697-04.2005.

[101] M.X. Cohen, C.E. Elger, B. Weber, Amygdala tractography predicts functional connectivity and learning during feedback-guided decision-making., Neuroimage. 39 (2008) 1396-1407. doi:10.1016/j.neuroimage.2007.10.004.

[102] J.Y. Davidow, K. Foerde, A. Galván, D. Shohamy, An upside to reward sensitivity: the hippocampus supports enhanced reinforcement learning in adolescence., Neuron. 92 (2016) 93-99. doi:10.1016/j.neuron.2016.08.031.

[103] O. Sporns, Network attributes for segregation and integration in the human brain., Curr. Opin. Neurobiol. 23 (2013) 162-171. doi:10.1016/j.conb.2012.11.015.

[104] J. Kube, D. Mathar, A. Horstmann, S.A. Kotz, A. Villringer, J. Neumann, Altered monetary loss processing and reinforcement-based learning in individuals with obesity., Brain Imaging Behav. 12 (2018) 1431-1449. doi:10.1007/s11682-017-9786-8.

[105] P.M. Johnson, P.J. Kenny, Dopamine D2 receptors in addiction-like reward dysfunction and compulsive eating in obese rats., Nat. Neurosci. 13 (2010) 635-641. doi:10.1038/nn.2519.

[106] P. Geha, G. Cecchi, R. Todd Constable, C. Abdallah, D.M. Small, Reorganization of brain connectivity in obesity., Hum. Brain Mapp. 38 (2017) 1403-1420. doi:10.1002/hbm.23462.

[107] M.-A. Cornier, K.L. McFadden, E.A. Thomas, J.L. Bechtell, L.S. Eichman, D.H. Bessesen, et al., Differences in the neuronal response to food in obesity-resistant as compared to obesity-prone individuals., Physiol. Behav. 110-111 (2013) 122-128. doi:10.1016/j.physbeh.2013.01.002.

[108] J. Garrison, B. Erdeniz, J. Done, Prediction error in reinforcement learning: a metaanalysis of neuroimaging studies., Neurosci. Biobehav. Rev. 37 (2013) 1297-1310. doi:10.1016/j.neubiorev.2013.03.023.

[109] P. Cáceres, R. San Martín, Low Cognitive Impulsivity Is Associated with Better Gain and Loss Learning in a Probabilistic Decision-Making Task., Front. Psychol. 8 (2017) 204. doi:10.3389/fpsyg.2017.00204.

[110] F. Del Missier, T. Mäntylä, P. Hansson, W. Bruine de Bruin, A.M. Parker, L.-G. Nilsson, The multifold relationship between memory and decision making: an individualdifferences study., J. Exp. Psychol. Learn. Mem. Cogn. 39 (2013) 1344-1364. doi:10.1037/a0032379.

[111] Y. Benjamini, Y. Hochberg, Controlling the false discovery rate: A practical and powerful approach to multiple testing, Journal of the Royal Statistical Society: Series B (Methodological). 57 (1995) 289-300. doi:10.1111/j.2517-6161.1995.tb02031.x. 


\section{SUPPLEMENTAL MATERIALS}

\section{S1. Probabilistic Selection Task Performance}

A summary of participant performance on the PST task training is presented in Table S1. Out of the 104 training trials in the modified Probabilistic Selection Task, participants responded to receive reinforcement on an average of $98.1 \pm 5.6$ trials. About half of those trials were reinforced with the reward (sweet taste, mean $=49.8 \pm 6.0$ trials), while participants also received a high number of punishment reinforcers (bitter taste, mean $=48.4 \pm 5.7$ trials). On the first block of training, choice accuracy (choose shape A, C, or E) was $50.0 \pm 10.0 \%$, and was the same $(50.0 \pm 10.0 \%)$ on the second block. On the third training block, accuracy was $49.0 \pm$ $9.0 \%$, then $52.0 \pm 11.0 \%$ on the final block (Figure S1). Participants did not show a statistically significant change in accuracy across the blocks $(F(1,338)=0.446 ; p=0.505)$. Additionally, there was no statistically significant difference in accuracy between the pairs $(F(2,252)=1.296$; $p=0.275)$. In a repeated measures ANOVA, controlling for within-participant effects, we found no significant difference in performance between pairs and over the blocks. On the PST posttest, participants chose shape A on $51.0 \pm 11.0 \%$ of trials, representing their $\%$ sensitivity to reward. Participants avoided shape B on $51.0 \pm 9.0 \%$ of trials, representing their $\%$ sensitivity to punishment. The correlation between sensitivity to reward and punishment was low $(r=0.135)$.

Table S1: PST Training Performance

\begin{tabular}{lccccc}
\hline & Run 1 & Run 2 & Run 3 & Run 4 & All Runs \\
\hline Number of Trials & 26 & 26 & 26 & 26 & 104 \\
Number of Responses (trials) & 23.86 & 24.93 & 25.15 & 24.20 & 98.14 \\
& $(1.55)$ & $(1.58)$ & $(1.80)$ & $(3.20)$ & $(5.59)$ \\
Response Time (s) & $1.60(0.01)$ & $1.59(0.01)$ & $1.59(0.01)$ & $1.59(0.01)$ & 1.59 \\
& & & & & $(0.005)$ \\
Percent Correct Overall & $0.50(0.10)$ & $0.49(0.10)$ & $0.49(0.09)$ & 0.52 & $0.5(0.05)$ \\
(accuracy) & & & & $(0.11)$ & \\
AB (80/20 Pair) & $0.52(0.18)$ & $0.50(0.16)$ & $0.50(0.15)$ & $0.51(0.18)$ & $0.51(0.07)$ \\
CD (70/30 Pair) & $0.49(0.16)$ & $0.51(0.18)$ & $0.50(0.17)$ & $0.51(0.18)$ & $0.50(0.09)$ \\
EF (60/40 Pair) & $0.49(0.20)$ & $0.48(0.19)$ & $0.47(0.16)$ & $0.52(0.20)$ & $0.49(0.09)$ \\
\hline
\end{tabular}


Figure S1: PST Training Performance

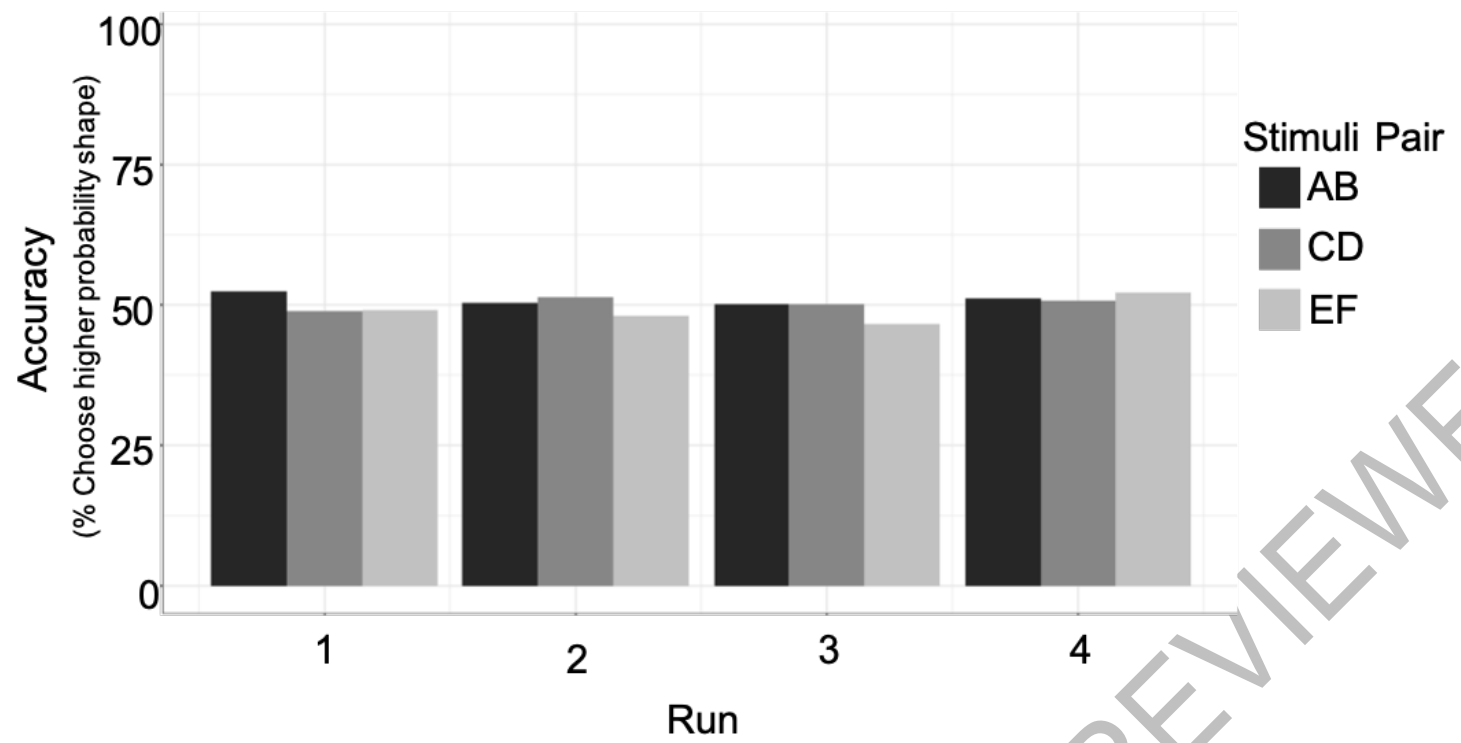

\section{S2. Associations between PST Task Performance and Participant Characteristics}

We tested for associations between posttest performance (sensitivity to reward and sensitivity to punishment) and participant characteristics including BMI, age, initial liking/wanting for the sweet and bitter tastes (as assessed via the prescan taste test), and performance during the PST training. We tested for associations with the continuous distribution of posttest performance, and also for differences between the "learner" and "non-learner" groups (split by average performance above or below chance). There were no significant associations with continuous performance or significant differences between posttest performance groups (Table S2).

Table S2: Associations between PST performance and Participant Characteristics

\begin{tabular}{lccccc}
\hline \multicolumn{1}{c}{ Characteristic } & $\begin{array}{c}\text { Correlation } \\
\text { with } \\
\text { Sensitivity to } \\
\text { Food } \\
\text { Reward }(r)\end{array}$ & $\begin{array}{c}\text { Correlation with } \\
\text { Sensitivity to } \\
\text { Food } \\
\text { Punishment } \\
(r)\end{array}$ & $\begin{array}{c}\text { Learners } \\
(\text { mean } \pm \text { SD) }\end{array}$ & $\begin{array}{c}\text { Non- } \\
\text { Learners } \\
\text { (mean } \pm \text { SD) }\end{array}$ & $\begin{array}{c}\text { Sig. Test } \\
(p \text {-value) }\end{array}$ \\
\hline BMl $\left(\mathrm{kg} / \mathrm{m}^{2}\right)$ & -0.127 & -0.021 & $24.3 \pm 3.1$ & $25.1 \pm 3.2$ & 0.29 \\
Age $(\mathrm{y})$ & 0.062 & 0.201 & $21.8 \pm 2.6$ & $21.1 \pm 2.0$ & 0.16 \\
Sweet Liking & -0.085 & 0.002 & $38.3 \pm 18.9$ & $39.2 \pm 19.5$ & 0.81 \\
Sweet Desire & -0.036 & 0.031 & $32.2 \pm 18.8$ & $29.7 \pm 23.7$ & 0.60 \\
Bitter Liking & -0.071 & 0.068 & $-47.5 \pm 24.1$ & $-43.2 \pm 27.8$ & 0.45 \\
Bitter Desire & -0.073 & 0.040 & $-51.6 \pm 25.4$ & $-44.4 \pm 30.0$ & 0.24 \\
Training Accuracy (\%) & 0.027 & 0.044 & $50.7 \pm 4.6$ & $49.8 \pm 4.7$ & 0.37 \\
Prediction error trials (\%) & 0.053 & -0.068 & $29.0 \pm 4.4$ & $30.6 \pm 4.5$ & 0.09 \\
\hline
\end{tabular}




\section{S3. Functional Connectivity Differences Between Reward and Punishment}

Significant differences between reward and punishment in functional connectivity (represented by $\mathrm{ROI}$ - ROI correlation) were identified via matrix t-test implemented in the 'psych' package (version 1.8.4) in the R statistical software package (version 3.5.1, R Foundation for Statistical Computing, Vienna, Austria). Significant differences in functional connectivity were considered using the Benjamini and Hochberg false discovery rate adjusted threshold of $p<0.05$ [111].

The difference in correlation value (reward $>$ punishment) is shown in Figure S2. Two correlations were significantly different between reward and punishment; 1) connectivity of the right intracalcarine cortex and left intracalcarine cortex, and 2) connectivity of the right oral somatosensory cortex and left oral somatosensory cortex. There were not significantly stronger correlations when comparing punishment $>$ reward. 
Figure S2: Differences in Functional Connectivity between Reward and Punishment

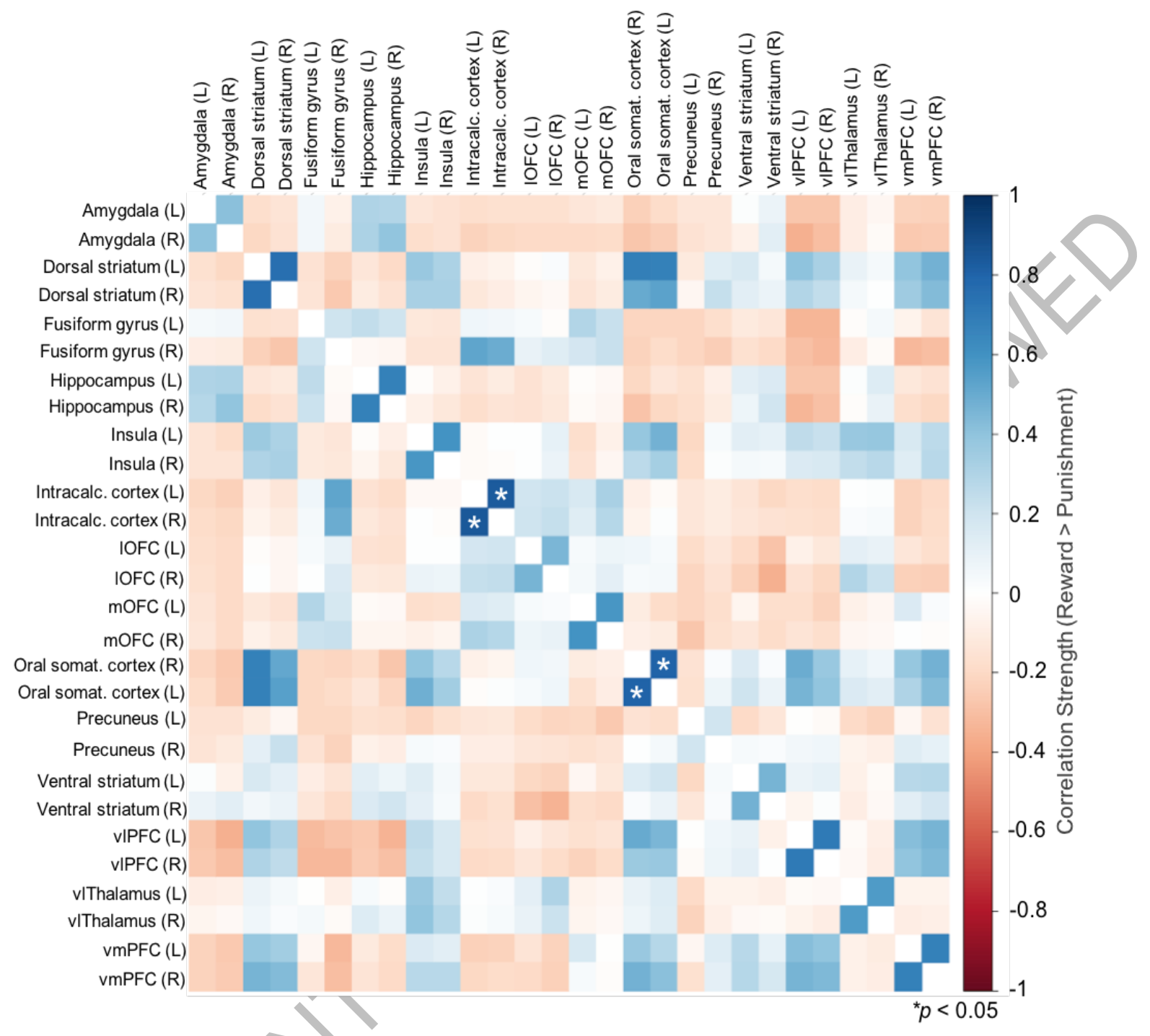

\section{S4. Measures of Community Topology}

To examine community integration, segregation, and hubness during the task, graph topology measures of participation coefficient, clustering coefficient, betweenness centrality were calculated for each community and then compared within the same condition. Community topology measures were not compared across condition because community membership was not the same in all condition for the majority of communities. Segregation of communities did not differ in choice, reward, or punishment. However, within condition, hubness and integration were significantly different between communities (Table S3). 
Table S3: Community Segregation, Integration, and Hubness Measures during Choice, Sweet Taste, and Bitter Taste

\begin{tabular}{|c|c|c|c|}
\hline Community & Choice $^{a}$ & Sweet $^{\text {a }}$ & Bitter $^{a}$ \\
\hline \multicolumn{4}{|c|}{ Segregation: Clustering Coefficient } \\
\hline 1 & $0.84(0.07)$ & $0.89(0.06)$ & $0.88(0.06)$ \\
\hline 2 & $0.84(0.08)$ & $0.87(0.06)$ & $0.87(0.06)$ \\
\hline 3 & $0.82(0.07)$ & $0.86(0.07)$ & $0.87(0.06)$ \\
\hline 4 & $0.84(0.08)$ & $0.87(0.07)$ & $0.86(0.06)$ \\
\hline 5 & $0.84(0.08)$ & $0.88(0.06)$ & $0.88(0.06)$ \\
\hline 6 & $0.82(0.08)$ & $0.87(0.06)$ & $0.87(0.06)$ \\
\hline 7 & $0.83(0.09)$ & $0.86(0.11)$ & $0.86(0.09)$ \\
\hline 8 & - & $0.89(0.06)$ & \\
\hline Significance Testing ${ }^{\mathrm{b}, \mathrm{c}}$ & $p=0.43$ & $p=0.75$ & $p=0.12$ \\
\hline \multicolumn{4}{|c|}{ Integration: Participation Coefficient } \\
\hline 1 & $0.77(0.23)$ & $0.83(0.17)$ & $0.87(0.1)$ \\
\hline 2 & $0.87(0.13)$ & $0.93(0.03)$ & $0.87(0.16)$ \\
\hline 3 & $0.84(0.16)$ & $0.91(0.05)$ & $0.89(0.09)$ \\
\hline 4 & $0.87(0.14)$ & $0.87(0.13)$ & $0.86(0.14)$ \\
\hline 5 & $0.80(0.20)$ & $0.92(0.05)$ & $0.89(0.12)$ \\
\hline 6 & $0.86(0.13)$ & $0.88(0.11)$ & $0.86(0.13)$ \\
\hline 7 & $0.64(0.24)$ & $0.62(0.26)$ & $0.59(0.29)$ \\
\hline 8 & - & $0.86(0.17)$ & - \\
\hline Significance Testing ${ }^{\mathrm{b}, \mathrm{c}}$ & $p=0.010^{*}$ & $p<0.0001^{*}$ & $p<0.0001^{*}$ \\
\hline \multicolumn{4}{|c|}{ Hubness: Betweenness Centrality } \\
\hline 1 & $0.009(0.005)$ & $0.007(0.005)$ & $0.007(0.004)$ \\
\hline 2 & $0.010(0.005)$ & $0.008(0.005)$ & $0.008(0.004)$ \\
\hline 3 & $0.011(0.005)$ & $0.009(0.005)$ & $0.009(0.005)$ \\
\hline 4 & $0.009(0.005)$ & $0.008(0.004)$ & $0.008(0.004)$ \\
\hline 5 & $0.009(0.006)$ & $0.007(0.005)$ & $0.007(0.004)$ \\
\hline 6 & $0.011(0.007)$ & $0.008(0.005)$ & $0.008(0.006)$ \\
\hline 7 & $0.005(0.004)$ & $0.003(0.003)$ & $0.004(0.005)$ \\
\hline 8 & - & $0.006(0.007)$ & - \\
\hline Significance Testing $\mathrm{b,c}$ & $p<0.0001^{*}$ & $p<0.0001^{*}$ & $p=0.0017^{*}$ \\
\hline
\end{tabular}

\section{S.5 Measures of Nodal Topology during Choice, Reward, and Punishment}

To examine differences in nodal integration, segregation, and hubness during the task, graph topology measures of participation coefficient, clustering coefficient, betweenness centrality were calculated. The sample's mean clustering coefficient, mean betweenness centrality, and mean participation coefficient during each condition are reported in Table S4. 
Table S4. Nodal Segregation, Integration, and Hubness Measures during Choice, Sweet Taste, and Bitter Taste

\begin{tabular}{|c|c|c|c|c|c|c|}
\hline ROls & \multicolumn{2}{|c|}{$\begin{array}{l}\text { Betweenness } \\
\text { Centrality } \\
\text { (mean \& SD) }\end{array}$} & \multicolumn{2}{|c|}{$\begin{array}{c}\text { Clustering } \\
\text { Coefficient } \\
\text { (mean \& SD) }\end{array}$} & \multicolumn{2}{|c|}{$\begin{array}{l}\text { Participation } \\
\text { Coefficient } \\
\text { (mean \& SD) }\end{array}$} \\
\hline Amygdala (L) & 0.008 & 0.006 & 0.84 & 0.09 & 0.84 & 0.13 \\
\hline Amygdala (R) & 0.007 & 0.006 & 0.85 & 0.09 & 0.81 & 0.17 \\
\hline Dorsal striatum (L) & 0.011 & 0.007 & 0.83 & 0.08 & 0.91 & 0.07 \\
\hline Dorsal striatum (R) & 0.011 & 0.008 & 0.83 & 0.09 & 0.92 & 0.06 \\
\hline Fusiform gyrus (L) & 0.012 & 0.010 & 0.82 & 0.09 & 0.87 & 0.14 \\
\hline Fusiform gyrus (R) & 0.011 & 0.007 & 0.82 & 0.08 & 0.86 & 0.14 \\
\hline Hippocampus (L) & 0.008 & 0.007 & 0.85 & 0.08 & 0.88 & 0.14 \\
\hline Hippocampus (R) & 0.008 & 0.009 & 0.85 & 0.09 & 0.86 & 0.12 \\
\hline Insula (L) & 0.008 & 0.007 & 0.85 & 0.09 & 0.88 & 0.15 \\
\hline Insula $(R)$ & 0.008 & 0.006 & 0.85 & 0.08 & 0.88 & 0.12 \\
\hline Intracalcarine cortex (L) & 0.011 & 0.007 & 0.82 & 0.08 & 0.89 & 0.09 \\
\hline Intracalcarine cortex $(\mathrm{R})$ & 0.011 & 0.007 & 0.82 & 0.08 & 0.86 & 0.16 \\
\hline Lateral OFC (L) & 0.011 & 0.008 & 0.82 & 0.09 & 0.83 & 0.19 \\
\hline Lateral OFC (R) & 0.012 & 0.008 & 0.82 & 0.09 & 0.89 & 0.11 \\
\hline Medial OFC (L) & 0.010 & 0.007 & 0.83 & 0.09 & 0.85 & 0.18 \\
\hline Medial OFC (R) & 0.010 & 0.007 & 0.83 & 0.08 & 0.85 & 0.17 \\
\hline Pre/postcentral gyrus (L) & 0.009 & 0.008 & 0.84 & 0.09 & 0.91 & 0.08 \\
\hline Pre/postcentral gyrus (R) & 0.010 & 0.007 & 0.84 & 0.08 & 0.90 & 0.13 \\
\hline Precuneus (L) & 0.007 & 0.006 & 0.84 & 0.09 & 0.83 & 0.14 \\
\hline Precuneus (R) & 0.010 & 0.009 & 0.84 & 0.09 & 0.86 & 0.15 \\
\hline Thalamus (L) & 0.010 & 0.008 & 0.83 & 0.09 & 0.90 & 0.09 \\
\hline Thalamus (R) & 0.010 & 0.009 & 0.83 & 0.09 & 0.89 & 0.12 \\
\hline Ventral striatum (L) & 0.005 & 0.006 & 0.83 & 0.11 & 0.65 & 0.26 \\
\hline Ventral striatum (R) & 0.005 & 0.005 & 0.83 & 0.10 & 0.65 & 0.30 \\
\hline Ventrolateral PFC (L) & 0.009 & 0.008 & 0.84 & 0.09 & 0.86 & 0.15 \\
\hline Ventrolateral PFC (R) & 0.009 & 0.008 & 0.84 & 0.09 & 0.86 & 0.13 \\
\hline Ventromedial PFC (L) & 0.011 & 0.008 & 0.83 & 0.09 & 0.88 & 0.14 \\
\hline \multirow[t]{2}{*}{ Ventromedial PFC (R) } & 0.008 & 0.007 & 0.85 & 0.09 & 0.85 & 0.18 \\
\hline & $\begin{array}{r}\text { Betwe } \\
\text { Cent } \\
\text { (mean }\end{array}$ & & $\begin{array}{l}\text { Sweet } \\
\text { Clust } \\
\text { Coeff } \\
\text { (mean }\end{array}$ & & $\begin{array}{r}\text { Partic } \\
\text { Coe } \\
\text { (mear }\end{array}$ & \\
\hline Amygdala (L) & 0.006 & 0.007 & 0.89 & 0.07 & 0.84 & 0.15 \\
\hline Amygdala (R) & 0.006 & 0.006 & 0.89 & 0.07 & 0.81 & 0.20 \\
\hline Dorsal striatum (L) & 0.009 & 0.006 & 0.86 & 0.07 & 0.93 & 0.03 \\
\hline Dorsal striatum (R) & 0.008 & 0.007 & 0.87 & 0.07 & 0.93 & 0.05 \\
\hline Fusiform gyrus (L) & 0.009 & 0.008 & 0.85 & 0.09 & 0.90 & 0.09 \\
\hline Fusiform gyrus (R) & 0.007 & 0.007 & 0.87 & 0.08 & 0.86 & 0.18 \\
\hline
\end{tabular}




\begin{tabular}{|c|c|c|c|c|c|c|}
\hline Hippocampus (L) & 0.008 & 0.008 & 0.88 & 0.07 & 0.90 & 0.08 \\
\hline Hippocampus (R) & 0.007 & 0.008 & 0.88 & 0.07 & 0.88 & 0.08 \\
\hline Insula (L) & 0.005 & 0.004 & 0.90 & 0.06 & 0.92 & 0.05 \\
\hline Insula (R) & 0.008 & 0.007 & 0.87 & 0.08 & 0.91 & 0.07 \\
\hline Intracalcarine cortex (L) & 0.008 & 0.007 & 0.87 & 0.07 & 0.91 & 0.08 \\
\hline Intracalcarine cortex $(R)$ & 0.009 & 0.006 & 0.86 & 0.07 & 0.91 & 0.12 \\
\hline Lateral OFC (L) & 0.008 & 0.006 & 0.87 & 0.07 & 0.90 & 0.08 \\
\hline Lateral OFC (R) & 0.009 & 0.007 & 0.87 & 0.08 & 0.89 & 0.13 \\
\hline Medial OFC (L) & 0.009 & 0.008 & 0.86 & 0.07 & 0.91 & 0.05 \\
\hline Medial OFC (R) & 0.009 & 0.007 & 0.86 & 0.08 & 0.91 & 0.08 \\
\hline Pre/postcentral gyrus (L) & 0.007 & 0.005 & 0.88 & 0.07 & 0.92 & 0.07 \\
\hline Pre/postcentral gyrus (R) & 0.008 & 0.007 & 0.87 & 0.07 & 0.93 & 0.03 \\
\hline Precuneus (L) & 0.007 & 0.006 & 0.88 & 0.07 & 0.87 & 0.11 \\
\hline Precuneus (R) & 0.009 & 0.011 & 0.87 & 0.07 & 0.89 & 0.10 \\
\hline Thalamus (L) & 0.007 & 0.006 & 0.88 & 0.07 & 0.92 & 0.04 \\
\hline Thalamus (R) & 0.008 & 0.012 & 0.87 & 0.08 & 0.92 & 0.06 \\
\hline Ventral striatum (L) & 0.003 & 0.003 & 0.87 & 0.09 & 0.69 & 0.24 \\
\hline Ventral striatum (R) & 0.004 & 0.004 & 0.85 & 0.16 & 0.63 & 0.29 \\
\hline Ventrolateral PFC (L) & 0.007 & 0.013 & 0.88 & 0.07 & 0.89 & 0.11 \\
\hline Ventrolateral PFC (R) & 0.005 & 0.005 & 0.89 & 0.06 & 0.86 & 0.18 \\
\hline Ventromedial PFC (L) & 0.009 & 0.008 & 0.86 & 0.07 & 0.91 & 0.09 \\
\hline Ventromedial PFC (R) & 0.007 & 0.006 & 0.88 & 0.07 & 0.90 & 0.13 \\
\hline ROls & \multicolumn{2}{|c|}{$\begin{array}{l}\text { Betweenness } \\
\text { Centrality } \\
\text { (mean \& SD) }\end{array}$} & \multicolumn{2}{|c|}{$\begin{array}{c}\text { Bitter Taste } \\
\text { Clustering } \\
\text { Coefficient } \\
\text { (mean \& SD) }\end{array}$} & \multicolumn{2}{|c|}{$\begin{array}{l}\text { Participation } \\
\text { Coefficient } \\
\text { (mean \& SD) }\end{array}$} \\
\hline Amygdala (L) & 0.007 & 0.009 & 0.88 & 0.07 & 0.85 & 0.15 \\
\hline Amygdala (R) & 0.005 & 0.005 & 0.90 & 0.07 & 0.85 & 0.11 \\
\hline Dorsal striatum (L) & 0.009 & 0.006 & 0.87 & 0.07 & 0.93 & 0.05 \\
\hline Dorsal striatum (R) & 0.009 & 0.008 & 0.87 & 0.07 & 0.92 & 0.05 \\
\hline Fusiform gyrus (L) & 0.009 & 0.008 & 0.87 & 0.07 & 0.89 & 0.12 \\
\hline Fusiform gyrus (R) & 0.008 & 0.007 & 0.87 & 0.07 & 0.85 & 0.16 \\
\hline Hippocampus (L) & 0.007 & 0.005 & 0.88 & 0.06 & 0.90 & 0.06 \\
\hline Hippocampus (R) & 0.007 & 0.007 & 0.88 & 0.07 & 0.89 & 0.09 \\
\hline Insula $(L)$ & 0.007 & 0.006 & 0.89 & 0.07 & 0.91 & 0.07 \\
\hline Insula (R) & 0.007 & 0.006 & 0.88 & 0.07 & 0.91 & 0.08 \\
\hline Intracalcarine cortex (L) & 0.009 & 0.007 & 0.86 & 0.07 & 0.90 & 0.11 \\
\hline Intracalcarine cortex $(R)$ & 0.009 & 0.006 & 0.86 & 0.07 & 0.91 & 0.09 \\
\hline Lateral OFC (L) & 0.007 & 0.006 & 0.87 & 0.07 & 0.90 & 0.08 \\
\hline Lateral OFC (R) & 0.010 & 0.008 & 0.86 & 0.08 & 0.90 & 0.10 \\
\hline Medial OFC (L) & 0.009 & 0.008 & 0.86 & 0.07 & 0.90 & 0.12 \\
\hline Medial OFC (R) & 0.008 & 0.005 & 0.87 & 0.07 & 0.90 & 0.10 \\
\hline Pre/postcentral gyrus (L) & 0.007 & 0.005 & 0.88 & 0.06 & 0.93 & 0.04 \\
\hline
\end{tabular}




\begin{tabular}{lllllll} 
Pre/postcentral gyrus (R) & 0.008 & 0.007 & 0.87 & 0.07 & 0.93 & 0.02 \\
Precuneus (L) & 0.007 & 0.006 & 0.88 & 0.06 & 0.85 & 0.17 \\
Precuneus (R) & 0.009 & 0.009 & 0.87 & 0.08 & 0.88 & 0.11 \\
Thalamus (L) & 0.007 & 0.006 & 0.87 & 0.07 & 0.91 & 0.07 \\
Thalamus (R) & 0.007 & 0.005 & 0.87 & 0.07 & 0.91 & 0.07 \\
Ventral striatum (L) & 0.005 & 0.009 & 0.86 & 0.09 & 0.69 & 0.25 \\
Ventral striatum (R) & 0.004 & 0.004 & 0.86 & 0.13 & 0.68 & 0.27 \\
Ventrolateral PFC (L) & 0.007 & 0.006 & 0.88 & 0.07 & 0.89 & 0.12 \\
Ventrolateral PFC (R) & 0.006 & 0.006 & 0.88 & 0.06 & 0.86 & 0.15 \\
Ventromedial PFC (L) & 0.009 & 0.007 & 0.86 & 0.07 & 0.92 & 0.06 \\
Ventromedial PFC (R) & 0.008 & 0.007 & 0.88 & 0.07 & 0.92 & 0.04 \\
\hline
\end{tabular}

\section{S.6 Network Organization and Network Topology Associated with Participant Characteristics and Behavioral Outcomes}

To test for associations between community segregation, integration and hubness with posttest performance and BMI, we correlated topology measures for each community with posttest performance ("choose reward" representing sensitivity to food reward, and "avoid punish" representing sensitivity to food punishment) and BMI. We found primarily weak correlations between topology measures and posttest performance/BMI. Adjusting for multiple comparisons, there was a significant positive correlation between posttest sensitivity to food punishment ("avoid punish") and segregation of choice community 1 (Table S5).

We also compared community organization between overweight/obese and healthy BMI groups, "learner" and "non-learner" posttest performance groups, and male and female participants. While we observed differences in community organization between groups in each condition, the majority of observed differences were not meaningfully different from network organization differences generated from random groups, as assessed by the normalized mutual information score (NMI; Figure S3). Values close to 0 indicate meaningful differences between the two groups. Of note, the observed NMI for community assignment of weight groups during choice was 1 SD below the mean NMI of the null distribution (denoted by *), while the observed NMI for community assignment of weight groups during sweet taste was 1 SD below the mean NMI of the null distribution. 
Table S5: Correlation of Segregation, Integration, and Hubness with Behavioral Outcomes

\begin{tabular}{|c|c|c|c|c|c|c|c|c|c|}
\hline & \multicolumn{3}{|c|}{ SEGREGATION } & \multicolumn{3}{|c|}{ INTEGRATION } & \multicolumn{3}{|c|}{ HUBNESS } \\
\hline & BMI & $\begin{array}{l}\text { Choose } \\
\text { Reward }\end{array}$ & $\begin{array}{l}\text { Avoid } \\
\text { Punish }\end{array}$ & BMI & $\begin{array}{l}\text { Choose } \\
\text { Reward }\end{array}$ & $\begin{array}{l}\text { Avoid } \\
\text { Punish }\end{array}$ & BMI & $\begin{array}{l}\text { Choose } \\
\text { Reward }\end{array}$ & $\begin{array}{l}\text { Avoid } \\
\text { Punish }\end{array}$ \\
\hline \multicolumn{10}{|c|}{$\begin{array}{l}\text { Choice } \\
\text { Community }\end{array}$} \\
\hline 1 & -0.056 & -0.129 & $0.289^{*}$ & 0.113 & 0.033 & 0.148 & 0.139 & 0.051 & -0.222 \\
\hline 2 & 0.195 & -0.237 & 0.228 & 0.115 & 0.033 & 0.107 & -0.139 & 0.217 & -0.235 \\
\hline 3 & 0.093 & -0.200 & 0.143 & -0.006 & -0.052 & -0.036 & 0.002 & 0.051 & -0.122 \\
\hline 4 & 0.098 & -0.239 & 0.185 & 0.054 & -0.202 & 0.089 & 0.040 & 0.221 & -0.073 \\
\hline 5 & 0.057 & -0.207 & 0.150 & 0.061 & -0.184 & 0.100 & 0.039 & 0.039 & -0.056 \\
\hline 6 & 0.166 & -0.215 & 0.097 & 0.043 & -0.010 & 0.146 & 0.047 & 0.136 & 0.021 \\
\hline 7 & 004 & -0.083 & 0.077 & -0.127 & -0.028 & -0.194 & -0.139 & & -0.060 \\
\hline \multicolumn{10}{|c|}{$\begin{array}{l}\text { Sweet } \\
\text { Community }\end{array}$} \\
\hline 1 & -0.132 & 0.057 & 0.176 & -0.083 & -0.180 & 0.025 & 0.185 & -0.066 & -0.131 \\
\hline 2 & 0.019 & 0.041 & 0.148 & -0.140 & 0.059 & 0.121 & 0.009 & -0.055 & -0.082 \\
\hline 3 & -0.089 & 0.054 & 0.147 & -0.160 & 0.139 & 0.012 & 0.012 & -0.043 & -0.002 \\
\hline 4 & -0.031 & 0.139 & 0.200 & -0.179 & -0.158 & 0.103 & 0.116 & -0.204 & -0.050 \\
\hline 5 & -0.052 & 0.002 & 0.168 & 0.048 & -0.057 & 0.022 & 0.056 & 0.033 & -0.185 \\
\hline 6 & -0.066 & 0.122 & 0.110 & -0.062 & 0.068 & -0.159 & 0.165 & -0.128 & -0.050 \\
\hline 7 & -0.052 & 0.014 & 0.134 & -0.088 & -0.032 & 0.094 & 0.181 & -0.103 & 0.037 \\
\hline 8 & -0.004 & -0.029 & 0.248 & 0.072 & -0.122 & 0.099 & -0.042 & 0.069 & -0.164 \\
\hline \multicolumn{10}{|c|}{$\begin{array}{l}\text { Bitter } \\
\text { Community }\end{array}$} \\
\hline 1 & 0.157 & 0.055 & 0.061 & -0.105 & 0.191 & 0.043 & -0.085 & -0.089 & -0.088 \\
\hline 2 & 0.081 & 0.016 & 0.045 & 0.025 & 0.073 & 0.113 & -0.020 & 0.014 & -0.081 \\
\hline 3 & 0.010 & 0.116 & 0.050 & 0.062 & -0.055 & -0.008 & 0.004 & -0.169 & -0.017 \\
\hline 4 & 0.094 & -0.007 & 0.053 & 0.071 & 0.044 & 0.019 & -0.015 & 0.083 & -0.058 \\
\hline 5 & 0.123 & 0.108 & -0.020 & -0.022 & -0.164 & -0.079 & -0.097 & -0.115 & 0.018 \\
\hline 6 & 0.168 & 0.052 & 0.014 & 0.086 & -0.049 & 0.139 & -0.053 & -0.096 & 0.087 \\
\hline & .061 & 0.121 & 0.047 & 0.182 & 0.194 & 0.105 & -0.060 & -0.152 & -0.018 \\
\hline
\end{tabular}

* significant correlation ( $\mathrm{p}$-value $<0.008)$ 
Figure S3: Observed NMI Scores Compared to Null Distribution

A) Male vs. Female
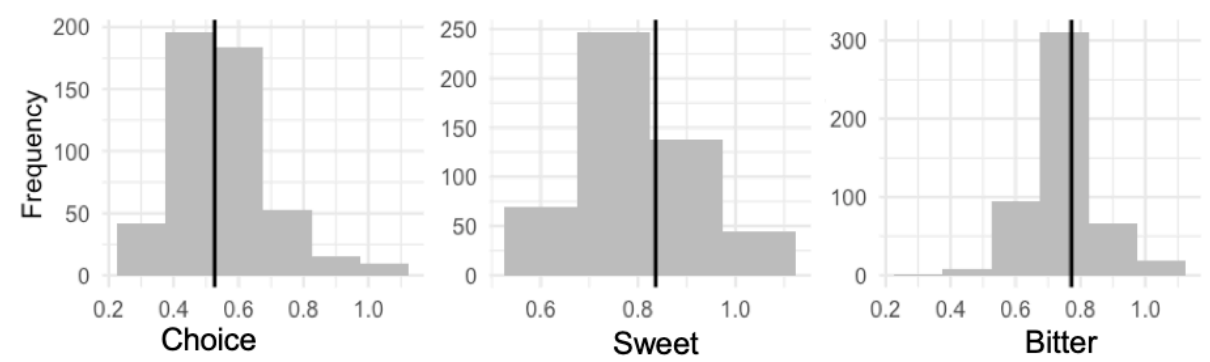

\section{B) Learners vs. Non-Learners}
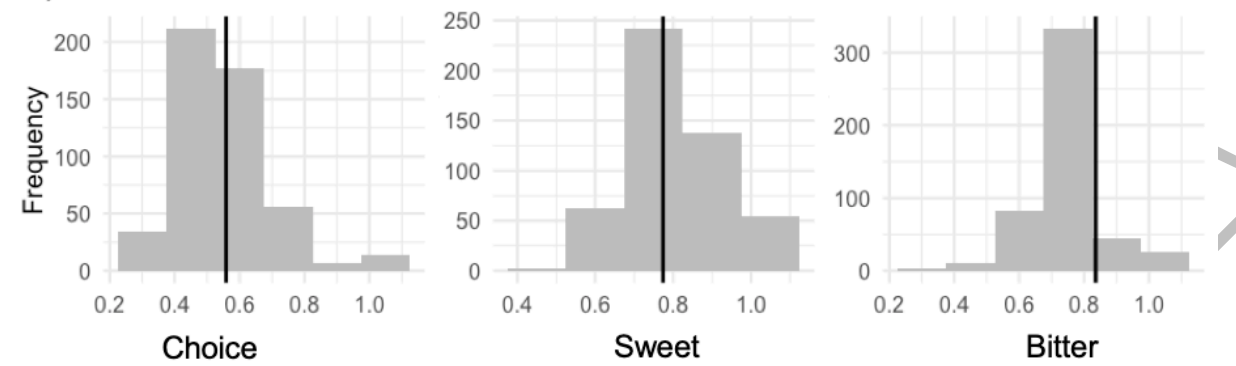

C) Normal Weight vs. Overweight/Obese
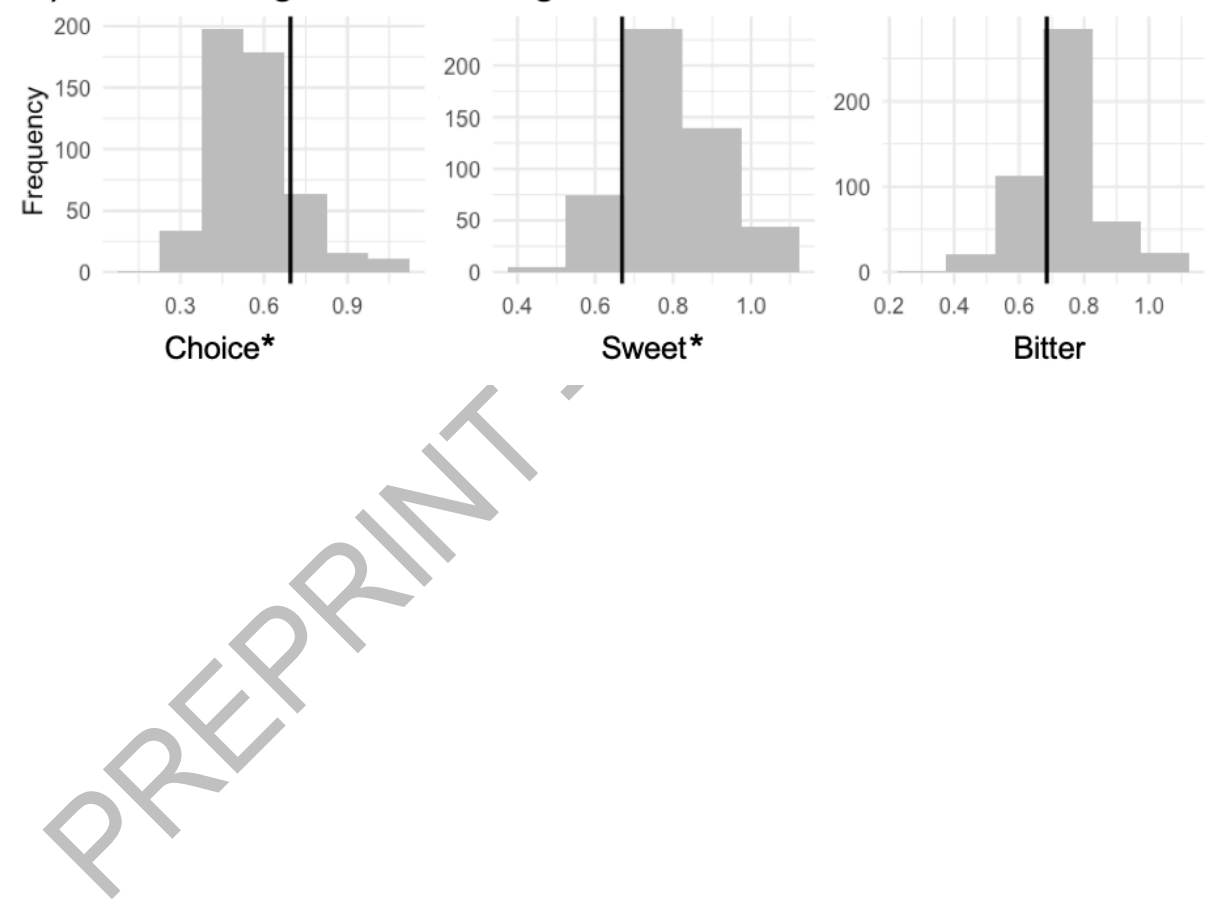